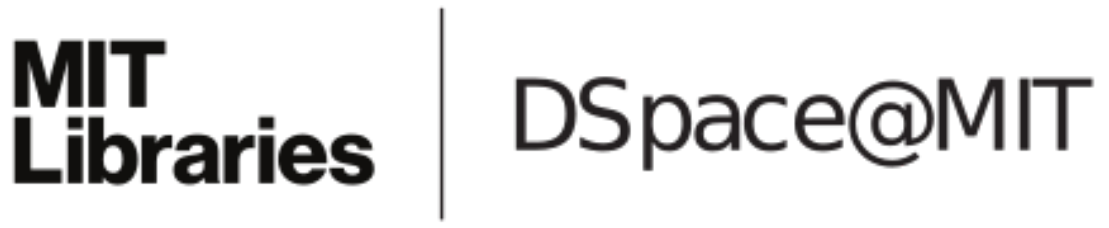

\author{
MIT Open Access Articles
}

\section{ASTROPHYSICAL IMPLICATIONS OF THE BINARY BLACK HOLE MERGER GW150914}

The MIT Faculty has made this article openly available. Please share how this access benefits you. Your story matters.

Citation: Abbott, B. P., R. Abbott, T. D. Abbott, M. R. Abernathy, F. Acernese, K. Ackley, C. Adams, et al. "ASTROPHYSICAL IMPLICATIONS OF THE BINARY BLACK HOLE MERGER GW150914." The Astrophysical Journal 818, no. 2 (February 11, 2016): L22.

As Published: http://dx.doi.org/10.3847/2041-8205/818/2//22

Publisher: Institute of Physics Publishing (IOP)

Persistent URL: http://hdl.handle.net/1721.1/102859

Version: Final published version: final published article, as it appeared in a journal, conference proceedings, or other formally published context

Terms of Use: Article is made available in accordance with the publisher's policy and may be subject to US copyright law. Please refer to the publisher's site for terms of use. 


\section{ASTROPHYSICAL IMPLICATIONS OF THE BINARY BLACK HOLE MERGER GW150914}

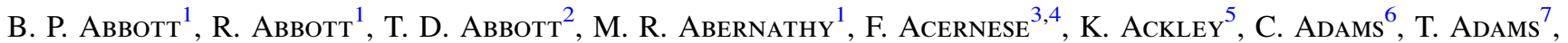

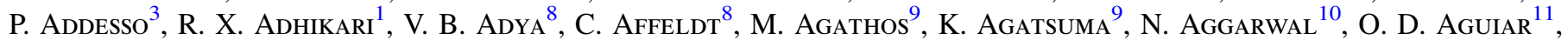
L. Aiello ${ }^{12,13}$, A. Ain ${ }^{14}$, P. Ajith ${ }^{15}$, B. Allen ${ }^{8,16,17}$, A. Allocca ${ }^{18,19}$, P. A. Altin ${ }^{20}$, S. B. Anderson ${ }^{1}$, W. G. Anderson ${ }^{16}$, K. Arai ${ }^{1}$, M. C. Araya ${ }^{1}$, C. C. Arceneaud ${ }^{21}$, J. S. Areeda ${ }^{22}$, N. Arnaud ${ }^{23}$, K. G. Arun ${ }^{24}$, S. Ascenzi ${ }^{13,25}$, G. Ashton ${ }^{26}$, M. Ast ${ }^{27}$, S. M. Aston ${ }^{6}$, P. Astone ${ }^{28}$, P. Aufmuth ${ }^{8}$, C. Aulbert ${ }^{8}$, S. BabaK ${ }^{29}$, P. Bacon ${ }^{30}$, M. K. M. Bader ${ }^{9}$, P. T. BaKer ${ }^{31}$, F. Baldaccini ${ }^{32,33}$, G. Ballardin ${ }^{34}$, S. W. Ballmer ${ }^{35}$, J. C. Barayoga ${ }^{1}$, S. E. Barclay ${ }^{36}$, B. C. Barish ${ }^{1}$, D. Barker ${ }^{37}$, F. Barone ${ }^{3,4}$, B. Barr ${ }^{36}$, L. Barsotti ${ }^{10}$, M. Barsuglia ${ }^{30}$, D. Barta ${ }^{38}$, J. Bartlett ${ }^{37}$, I. Bartos ${ }^{39}$, R. Bassiri ${ }^{40}$, A. Basti ${ }^{18,19}$, J. C. Batch ${ }^{37}$, C. Baune ${ }^{8}$, V. Bavigadda ${ }^{34}$, M. Bazzan ${ }^{41,42}$, B. Behnke ${ }^{29}$, M. Bejger ${ }^{43}$, C. Belczynski ${ }^{44}$, A. S. Bell ${ }^{36}$,

C. J. Bell ${ }^{36}$, B. K. Berger ${ }^{1}$, J. Bergman ${ }^{37}$, G. BergmanN ${ }^{8}$, C. P. L. Berry ${ }^{45}$, D. Bersanetti ${ }^{46,47}$, A. Bertolini ${ }^{9}$,

J. Betzwieser ${ }^{6}$, S. Bhagwat ${ }^{35}$, R. Bhandare ${ }^{48}$, I. A. Bilenko ${ }^{49}$, G. Billingsley ${ }^{1}$, J. Birch ${ }^{6}$, R. Birney ${ }^{50}$, S. Biscans $^{10}$, A. Bisht ${ }^{8,17}$, M. Bitossi ${ }^{34}$, C. Biwer ${ }^{35}$, M. A. Bizouard ${ }^{23}$, J. K. Blackburn ${ }^{1}$, C. D. Blair ${ }^{51}$, D. G. Blair ${ }^{51}$, R. M. Blair ${ }^{37}$,

S. Bloemen ${ }^{52}$, O. Bock ${ }^{8}$, T. P. Bodiya ${ }^{10}$, M. Boer ${ }^{53}$, G. Bogaert ${ }^{53}$, C. BogaN ${ }^{8}$, A. Bohe ${ }^{29}$, P. Bojtos ${ }^{54}$, C. Bond $^{45}$,

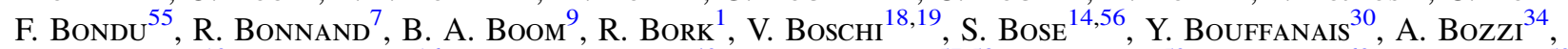

C. Bradaschia ${ }^{19}$, P. R. Brady $^{16}$, V. B. Braginsky ${ }^{49}$, M. Branchesi ${ }^{57,58}$, J. E. Brau ${ }^{59}$, T. Briant ${ }^{60}$, A. Brillet ${ }^{53}$, M. Brinkmann ${ }^{8}$, V. Brisson ${ }^{23}$, P. Brockill ${ }^{16}$, A. F. Brooks ${ }^{1}$, D. A. Brown ${ }^{35}$, D. D. Brown ${ }^{45}$, N. M. Brown ${ }^{10}$,

C. C. Buchanan ${ }^{2}$, A. Buikema ${ }^{10}$, T. Bulik ${ }^{44}$, H. J. Bulten ${ }^{9,61}$, A. Buonanno ${ }^{29,62}$, D. Buskulic ${ }^{7}$, C. Buy ${ }^{30}$, R. L. Byer ${ }^{40}$,

L. Cadonati $^{63}$, G. Cagnoli ${ }^{64,65}$, C. Cahillane ${ }^{1}$, J. Calderón Bustillo ${ }^{63,66}$, T. Callister ${ }^{1}$, E. Calloni ${ }^{4,67}$, J. B. Camp ${ }^{68}$,

K. C. Cannon ${ }^{69}$, J. CaO ${ }^{70}$, C. D. Capano ${ }^{8}$, E. Capocasa ${ }^{30}$, F. Carbognani ${ }^{34}$, S. Caride ${ }^{71}$, J. Casanulva Diaz ${ }^{23}$,

C. Casentini ${ }^{13,25}$, S. Caudill ${ }^{16}$, M. Cavaglià ${ }^{21}$, F. Cavalier ${ }^{23}$, R. Cavalieri ${ }^{34}$, G. Cella ${ }^{19}$, C. Cepeda $^{1}$,

L. Cerboni Baiardi ${ }^{57,58}$, G. Cerretani ${ }^{18,19}$, E. Cesarini ${ }^{13,25}$, R. Chakraborty ${ }^{1}$, T. Chalermsongsak ${ }^{1}$, S. J. Chamberlin ${ }^{72}$,

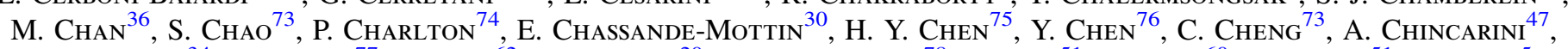
A. Сhiummo ${ }^{34}$, H. S. $\mathrm{Cho}^{77}$, M. $\mathrm{Cho}^{62}$, J. H. $\mathrm{Chow}^{20}$, N. Christensen $^{78}$, Q. $\mathrm{Chu}^{51}$, S. Chua ${ }^{60}$, S. ChunG ${ }^{51}$, G. Ciani ${ }^{5}$, F. Clara $^{37}$, J. A. Clark ${ }^{63}$, F. Cleva ${ }^{53}$, E. Coccia ${ }^{12,13,25}$, P.-F. Cohadon $^{60}$, A. Colla $^{28,79}$, C. G. Collette ${ }^{80}$, L. Cominsky ${ }^{81}$, M. Constancio JR. ${ }^{11}$, A. Conte 28,79 , L. Conti ${ }^{42}$, D. Cook $^{37}$, T. R. Corbitt $^{2}$, N. Cornish ${ }^{31}$, A. Corsi ${ }^{82}$, S. Cortese ${ }^{34}$, C. A. $\operatorname{Costa}^{11}$, M. W. Coughlin $^{78}$, S. B. Coughlin ${ }^{83}$, J.-P. Coulon ${ }^{53}$, S. T. Countryman ${ }^{39}$, P. Couvares ${ }^{1}$, E. E. Cowan ${ }^{63}$, D. M. Coward ${ }^{51}$, M. J. Cowart ${ }^{6}$, D. C. Coyne ${ }^{1}$, R. Coyne ${ }^{82}$, K. Craig ${ }^{36}$, J. D. E. Creighton ${ }^{16}$, J. Cripe ${ }^{2}$, S. G. Crowder ${ }^{84}$,

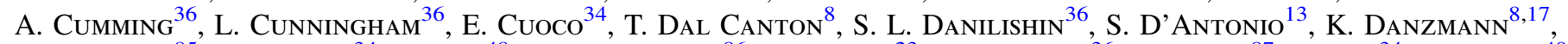
N. S. Darman ${ }^{85}$, V. Dattilo ${ }^{34}$, I. Dave ${ }^{48}$, H. P. Daveloza ${ }^{86}$, M. Davier ${ }^{23}$, G. S. Davies ${ }^{36}$, E. J. Daw ${ }^{87}$, R. DaY Di $^{34}$, D. DeBra ${ }^{40}$, G. Debreczeni ${ }^{38}$, J. Degallatix ${ }^{65}$, M. De Laurentis, 47 , S. Deléglise ${ }^{60}$, W. Del Pozzo ${ }^{45}$, T. Denker ${ }^{8,17}$, T. Dent ${ }^{8}$,

H. Dereli ${ }^{53}$, V. Dergachev ${ }^{1}$, R. DeRosa ${ }^{6}$, R. T. DeRosa ${ }^{4,67}$, R. DeSalvo $^{88}$, S. Dhurandhar ${ }^{14}$, M. C. Díaz ${ }^{86}$, L. Di Fiore ${ }^{4}$, M. Di Giovanni 28,79 , A. Di Lieto ${ }^{18,19}$, S. Di PaCe ${ }^{28,79}$, I. Di Palma ${ }^{8,29}$, A. Di Virgilio ${ }^{19}$, G. Dojcinoski ${ }^{89}$, V. Dolique ${ }^{65}$,

F. Donovan ${ }^{10}$, K. L. Dooley ${ }^{21}$, S. Doravari ${ }^{6,8}$, R. Douglas ${ }^{36}$, T. P. Downes ${ }^{16}$, M. Drago ${ }^{8,90,91}$, R. W. P. Drever ${ }^{1}$,

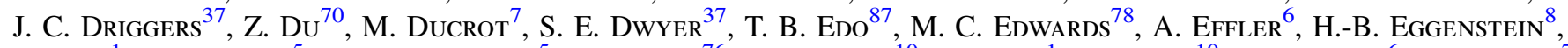

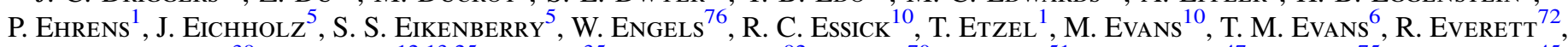
M. FACTOUROVICH ${ }^{39}$, V. FAFONE ${ }^{12,13,25}$, H. FAIR ${ }^{35}$, S. FAIRHURST ${ }^{92}$, X. FAN ${ }^{70}$, Q. FANG ${ }^{51}$, S. FARINON ${ }^{47}$, B. FARR ${ }^{75}$, W. M. FARR ${ }^{45}$, M. Favata ${ }^{89}$, M. Fays ${ }^{92}$, H. Fehrmann ${ }^{8}$, M. M. Fejer ${ }^{40}$, I. Ferrante ${ }^{18,19}$, E. C. Ferreira ${ }^{11}$, F. Ferrini ${ }^{34}$, F. Fidecaro ${ }^{18,19}$,

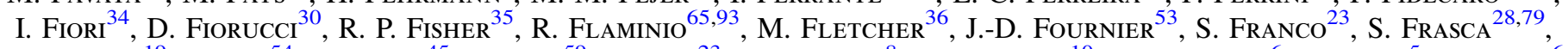

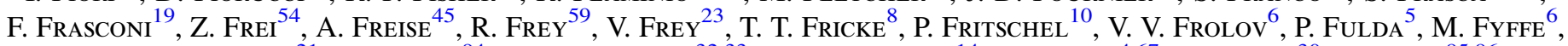
H. A. G. Gabbard ${ }^{21}$, J. R. Gair ${ }^{94}$, L. Gammaitoni ${ }^{32,33}$, S. G. GaOnKar ${ }^{14}$, F. Garufi ${ }^{4,67}$, A. Gatto ${ }^{30}$ G. Gaur $^{95,96}$,

N. Gehrels ${ }^{68}$, G. Gemme ${ }^{47}$, B. Gendre ${ }^{53}$, E. Genin ${ }^{34}$, A. Gennai ${ }^{19}$, J. George ${ }^{48}$, L. Gergely ${ }^{97}$, V. Germain ${ }^{7}$,

Archisman Ghosh ${ }^{15}$, S. Ghosh ${ }^{9,52}$, J. A. Giaime ${ }^{2,6}$, K. D. Giardina ${ }^{6}$, A. Giazotto ${ }^{19}$, K. Gill ${ }^{98}$, A. GlaEfKe ${ }^{36}$, E. Goetz $^{71}$, R. Goetz ${ }^{5}$, L. Gondan ${ }^{54}$, G. González ${ }^{2}$, J. M. Gonzalez Castro ${ }^{18,19}$, A. GopaKumar ${ }^{99}$, N. A. Gordon ${ }^{36}$,

M. L. Gorodetsky ${ }^{49}$, S. E. Gossan ${ }^{1}$, M. Gosselin ${ }^{34}$, R. Gounty ${ }^{7}$, C. Graef ${ }^{36}$, P. B. GrafF ${ }^{62}$, M. Granata ${ }^{65}$ A. Grant $^{36}$, S. Gras ${ }^{10}$, C. GraY ${ }^{37}$, G. Greco ${ }^{57,58}$, A. C. Green ${ }^{45}$, P. Groot $^{52}$, H. Grote ${ }^{8}$, S. Grunewald ${ }^{29}$, G. M. Guidi ${ }^{57,58}$, X. Guo ${ }^{70}$, A. Gupta ${ }^{14}$, M. K. Gupta ${ }^{96}$, K. E. Gushwa ${ }^{1}$, E. K. Gustafson ${ }^{1}$, R. Gustafson ${ }^{71}$, J. J. HaCKer ${ }^{22}$, B. R. Hall ${ }^{56}$, E. D. Hall ${ }^{1}$,

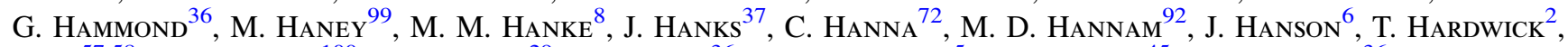
J. Harms ${ }^{57,58}$, G. M. Harry ${ }^{100}$, I. W. Harry ${ }^{29}$, M. J. Hart ${ }^{36}$, M. T. Hartman ${ }^{5}$, C.-J. Haster ${ }^{45}$, K. Haughian ${ }^{36}$, A. HeidmanN ${ }^{60}$, M. C. Heintze ${ }^{5,6}$, H. HeitmanN ${ }^{33}$, P. Hello ${ }^{23}$, G. Hemming ${ }^{34}$, M. Hendry ${ }^{36}$, I. S. Heng ${ }^{36}$, J. Hennig ${ }^{36}$, A. W. Heptonstall ${ }^{1}$,

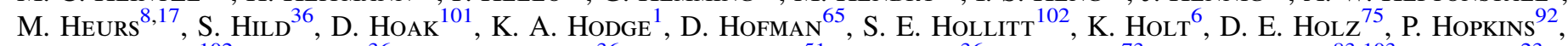

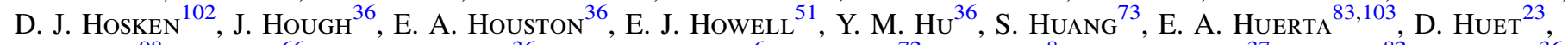

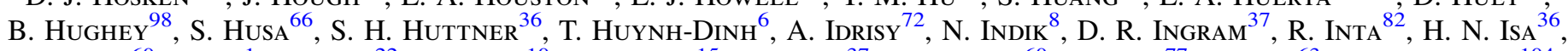

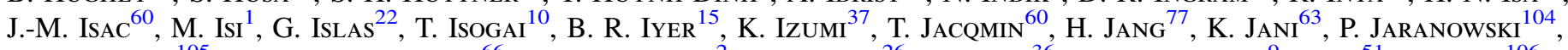

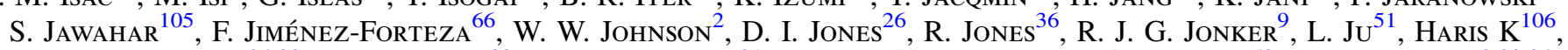
C. V. Kalaghatgi ${ }^{24,92}$, V. Kalogera ${ }^{83}$, S. Kandhasamy ${ }^{21}$, G. Kang ${ }^{77}$, J. B. Kanner ${ }^{1}$, S. Karki ${ }^{59}$, M. KasPrZaCK ${ }^{2,23,34}$, 
E. Katsavounidis ${ }^{10}$, W. Katzman $^{6}$, S. Kaufer ${ }^{17}$, T. Kaur ${ }^{51}$, K. Kawabe ${ }^{37}$, F. Kawazoe ${ }^{8}$, F. Kéfélian ${ }^{53}$, M. S. Kehl ${ }^{69}$, D. Keitel ${ }^{8,66}$, D. B. Kelley ${ }^{35}$, W. Kells ${ }^{1}$, R. Kennedy $^{87}$, J. S. KeY ${ }^{86}$, A. Khalaidovski ${ }^{8}$, F. Y. Khalili ${ }^{49}$, I. Khan ${ }^{12}$,

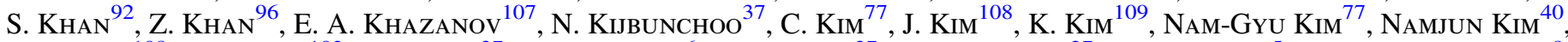

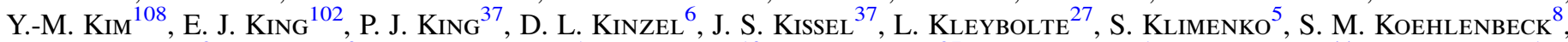
K. Kokeyama ${ }^{2}$, S. Koley ${ }^{9}$, V. Kondrashov ${ }^{1}$, A. Kontos ${ }^{10}$, M. Korobko ${ }^{27}$, W. Z. Korth ${ }^{1}$, I. Kowalska ${ }^{44}$, D. B. KozaK ${ }^{1}$,

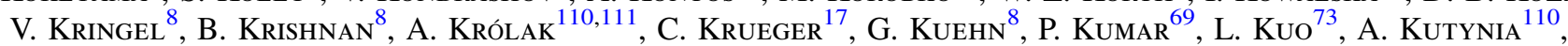

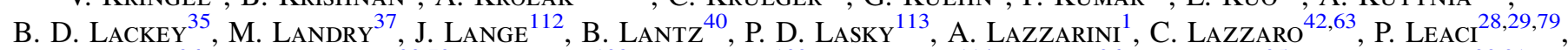

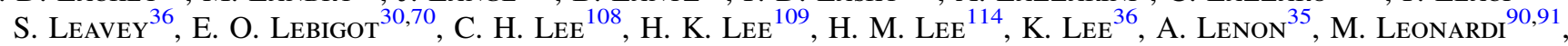

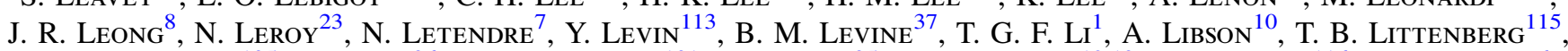

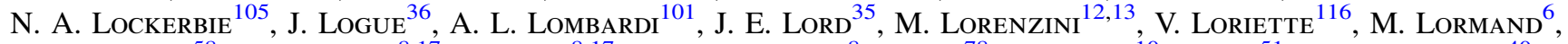

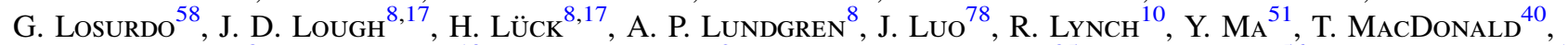

B. MachenschalK ${ }^{8}$, M. MacInnis ${ }^{10}$, D. M. Macleod ${ }^{2}$, F. Magaña-Sandoval ${ }^{35}$, R. M. MageE ${ }^{56}$, M. Mageswaran ${ }^{1}$, E. Majorana $^{28}$, I. MaKsimovic ${ }^{116}$, V. Malvezzi ${ }^{13,25}$, N. MAN ${ }^{53}$, I. Mandel ${ }^{45}$, V. Mandic ${ }^{84}$, V. Mangano ${ }^{36}$, G. L. Mansell ${ }^{20}$, M. Manske ${ }^{16}$, M. Mantovani ${ }^{34}$, F. Marchesoni ${ }^{33,117}$, F. Marion ${ }^{7}$, S. Márka ${ }^{39}$, Z. Márka ${ }^{39}$, A. S. Markosyan ${ }^{40}$, E. Maros $^{1}$,

F. Martelli ${ }^{57,58}$, L. Martellini ${ }^{53}$, I. W. Martin ${ }^{36}$, R. M. Martin ${ }^{5}$, D. V. Martynovi ${ }^{1}$, J. N. Marx ${ }^{1}$, K. Mason $^{10}$,

A. Masserot ${ }^{7}$, T. J. Massinger ${ }^{35}$, M. Masso-Reid ${ }^{36}$, F. Matichard ${ }^{10}$, L. Matone $^{39}$, N. Mavalvala ${ }^{10}$, N. Mazumder ${ }^{56}$, G. Mazzolo $^{8}$, R. McCarthy ${ }^{37}$, D. E. McClelland ${ }^{20}$, S. McCormick ${ }^{6}$, S. C. McGuire ${ }^{118}$, G. McIntyre ${ }^{1}$, J. McIver ${ }^{101}$, D. J. McManus ${ }^{20}$, S. T. McWilliams ${ }^{103}$, D. Meacher ${ }^{72}$, G. D. Meadors ${ }^{8,29}$, J. Meidam ${ }^{9}$, A. Melatos ${ }^{85}$, G. Mendell ${ }^{37}$, D. Mendoza-Gandara ${ }^{8}$, R. A. Mercer ${ }^{16}$, E. Merill ${ }^{37}$, M. Merzougur $^{53}$, S. Meshrov ${ }^{1}$, C. Messenger ${ }^{36}$, C. Messick ${ }^{72}$, P. M. Meyers ${ }^{84}$, F. Mezzani ${ }^{28,79}$, H. Miao ${ }^{45}$, C. Michel ${ }^{65}$, H. Middleton ${ }^{45}$, E. E. Mikhailov ${ }^{119}$, L. Milano ${ }^{4,67}$, J. MilleR ${ }^{10}$, M. Millhouse ${ }^{31}$, Y. Minenkov ${ }^{13}$, J. Ming ${ }^{8,29}$, S. MirsheKari ${ }^{120}$, C. Mishra ${ }^{15}$, S. Mitra ${ }^{14}$, V. P. Mitrofanov ${ }^{49}$, G. Mitselmakher ${ }^{5}$, R. Mittleman $^{10}$, A. Moggi ${ }^{19}$, M. Mohan $^{34}$, S. R. P. Mohapatra ${ }^{10}$, M. Montani ${ }^{57,58}$, B. C. Moore ${ }^{89}$, C. J. Moore ${ }^{121}$, D. Moraru ${ }^{37}$, G. Moreno ${ }^{37}$, S. R. Morriss ${ }^{86}$, K. Mossavi ${ }^{8}$, B. Mours ${ }^{7}$, C. M. Mow-Lowry ${ }^{45}$, C. L. Mueller ${ }^{5}$, G. Mueller ${ }^{5}$, A. W. Muir ${ }^{92}$, Arunava Mukherjee ${ }^{15}$, D. Mukherjee ${ }^{16}$, S. Mukherjee ${ }^{86}$, N. Mukund ${ }^{14}$, A. Mullavey ${ }^{6}$, J. Munch ${ }^{102}$, D. J. Murphy ${ }^{39}$, P. G. Murray ${ }^{36}$, A. Mytidis ${ }^{5}$, I. NARdecchia ${ }^{13,25}$, L. NaticChioni ${ }^{28,79}$, R. K. NayaK ${ }^{122}$, V. Necula ${ }^{5}$, K. Nedkova ${ }^{101}$, G. Nelemans ${ }^{9,52}$, M. Neri ${ }^{46,47}$, A. Neunzert $^{71}$, G. Newton $^{36}$, T. T. NguYen ${ }^{20}$, A. B. Nielsen ${ }^{8}$,

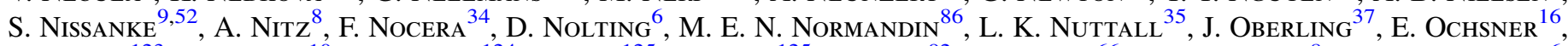

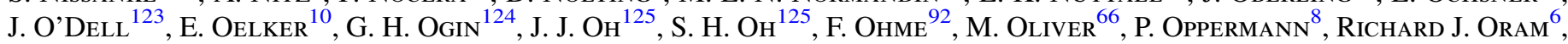
B. O’Reilly ${ }^{6}$, R. O’Shaughnessy ${ }^{112}$, D. J. OtTawaY ${ }^{102}$, R. S. Ottens ${ }^{5}$, H. Overmier ${ }^{6}$, B. J. Owen ${ }^{82}$, A. PaI ${ }^{106}$, S. A. PaI ${ }^{48}$, J. R. Palamos ${ }^{59}$, O. Palashov ${ }^{107}$, C. Palomba ${ }^{28}$, A. Pal-Singh ${ }^{27}$, H. Pan ${ }^{73}$, C. Pankow ${ }^{83}$, F. Pannarale ${ }^{92}$, B. C. Pant ${ }^{48}$, F. Paoletti ${ }^{19,34}$, A. Paoli ${ }^{34}$, M. A. Papa ${ }^{8,16,29}$, H. R. Paris ${ }^{40}$, W. Parker ${ }^{6}$, D. Pascucci ${ }^{36}$, A. Pasqualetti ${ }^{34}$,

R. Passaquieti ${ }^{18,19}$, D. Passuello ${ }^{19}$, B. Patricellit ${ }^{18,19}$, Z. Patrick ${ }^{40}$, B. L. Pearlstone ${ }^{36}$, M. Pedraza ${ }^{1}$, R. Pedurand ${ }^{65}$,

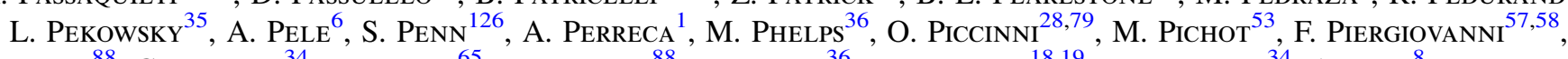
V. Pierro ${ }^{88}$, G. Pillant ${ }^{34}$, L. Pinard ${ }^{65}$, I. M. Pinto ${ }^{88}$, M. Pitkin ${ }^{36}$, R. Poggiani ${ }^{18,19}$, P. Popolizio ${ }^{34}$, A. Post ${ }^{8}$, J. Powell ${ }^{36}$, J. Prasad ${ }^{14}$, V. Predoi ${ }^{92}$, S. S. Premachandra ${ }^{113}$, T. Prestegard ${ }^{84}$, L. R. Price ${ }^{1}$, M. Prijatelj ${ }^{34}$, M. Principe ${ }^{88}$, S. Privitera ${ }^{29}$, R. Prix ${ }^{8}$, G. A. Prodi ${ }^{90,91}$, L. Prokhorov ${ }^{49}$, O. Puncken ${ }^{8}$, M. Punturo $^{33}$, P. Puppo ${ }^{28}$, M. PÜrrer ${ }^{29}$, H. Qi $^{16}$,

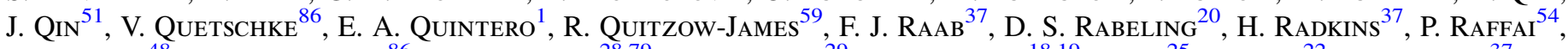

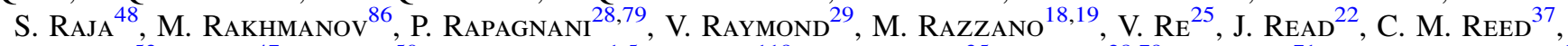

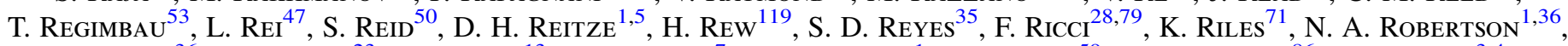

R. Robie ${ }^{36}$, F. Robinet ${ }^{23}$, A. Rocchi ${ }^{13}$, L. Rolland ${ }^{7}$, J. G. Rollins ${ }^{1}$, V. J. Roma ${ }^{59}$, J. D. Romano ${ }^{86}$, R. Romano ${ }^{3,4}$,

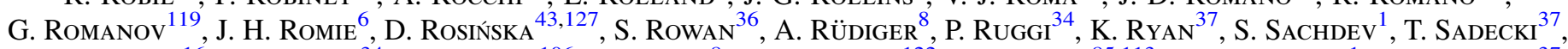

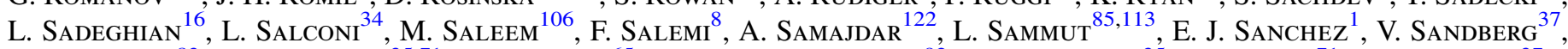
B. Sandeen ${ }^{83}$, J. R. Sanders ${ }^{35,71}$, B. Sassolas ${ }^{65}$, B. S. SathyapraKash ${ }^{92}$, P. R. Saulson ${ }^{35}$, O. Sauter ${ }^{71}$, R. L. Savage ${ }^{37}$,

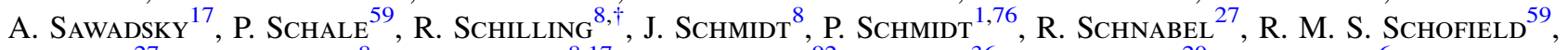

A. Schönbeck ${ }^{27}$, E. Schreiber ${ }^{8}$, D. Schuette ${ }^{8,17}$, B. F. Schutz ${ }^{92}$, J. Scotт ${ }^{36}$, S. M. Scott $^{20}$, D. Sellers ${ }^{6}$, D. Sentenac ${ }^{34}$, V. Sequino ${ }^{13,25}$, A. Sergeev ${ }^{107}$, G. Serna ${ }^{22}$, Y. Setyawati ${ }^{9,52}$, A. Sevigny ${ }^{37}$, D. A. Shaddock ${ }^{20}$, S. Shah ${ }^{9,52}$, M. S. Shahriar ${ }^{83}$, M. Shaltev ${ }^{8}$, Z. Shao ${ }^{1}$, B. Shapiro ${ }^{40}$, P. Shawhan $^{62}$, A. ShePerd ${ }^{16}$, D. H. Shoemaker ${ }^{10}$, D. M. Shoemaker ${ }^{63}$, K. Siellez ${ }^{53,63}$, X. Siemens ${ }^{16}$, D. Sigg ${ }^{37}$, A. D. Silva ${ }^{11}$, D. Simakov ${ }^{8}$, A. Singer ${ }^{1}$, L. P. Singer ${ }^{68}$, A. Singh $^{8,29}$, R. Singh ${ }^{2}$, A. Singhal ${ }^{12}$, A. M. Sintes ${ }^{66}$, B. J. J. Slagmolen ${ }^{20}$, J. R. Smith ${ }^{22}$, N. D. Smith ${ }^{1}$, R. J. E. Smith ${ }^{1}$, E. J. Son ${ }^{125}$, B. Sorazu ${ }^{36}$, F. Sorrentino ${ }^{47}$, T. Souradeep ${ }^{14}$, A. K. Srivastava ${ }^{96}$, A. Staley ${ }^{39}$, M. Steinke ${ }^{8}$,

J. Steinlechner ${ }^{36}$, S. Steinlechner ${ }^{36}$, D. SteinmeYer ${ }^{8,17}$, B. C. StePhens ${ }^{16}$, S. P. Stevenson ${ }^{45}$, R. Stone ${ }^{86}$, K. A. Strain ${ }^{36}$, N. Straniero ${ }^{65}$, G. Stratta ${ }^{57,58}$, N. A. Strauss ${ }^{78}$, S. Strigin ${ }^{49}$, R. Sturani ${ }^{120}$, A. L. Stuver ${ }^{6}$, T. Z. Summerscales ${ }^{128}$,

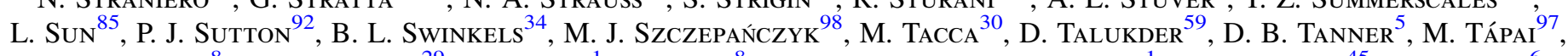

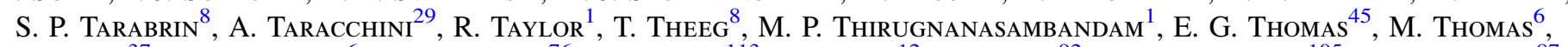
P. Thomas ${ }^{37}$, K. A. Thorne ${ }^{6}$, K. S. Thorne ${ }^{76}$, E. Thrane ${ }^{113}$, S. Tiwari ${ }^{12}$, V. Tiwari ${ }^{92}$, K. V. Tokmakov ${ }^{105}$, C. Tomlinson $^{87}$, M. Tonelli ${ }^{18,19}$, C. V. Torres ${ }^{86, \ddagger}$, C. I. Torrie ${ }^{1}$, D. Törrä ${ }^{45}$, F. Travasso ${ }^{32,33}$, G. TraYlor ${ }^{6}$, D. Trifirò ${ }^{21}$, M. C. Tringalit ${ }^{90,91}$,

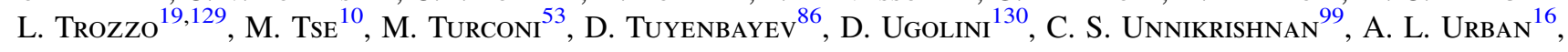


S. A. Usman ${ }^{35}$, H. Vahlbruch ${ }^{17}$, G. Vajente ${ }^{1}$, G. VAldes $^{86}$, N. van BaKel ${ }^{9}$, M. van Beuzekom ${ }^{9}$, J. F. J. van den BRand ${ }^{61,9}$, C. van den Broeck ${ }^{9}$, D. C. VAnder-Hyde ${ }^{22,35}$, L. van der SchaAf ${ }^{9}$, J. V. van Heijningen ${ }^{9}$, A. A. van VegGel ${ }^{36}$, M. Vardaro ${ }^{41,42}$, S. Vass ${ }^{1}$, M. Vasúth ${ }^{38}$, R. VAulin $^{10}$, A. Vecchio ${ }^{45}$, G. Vedovato $^{42}$, J. Veitch $^{45}$, P. J. Veitch ${ }^{102}$, K. Venkateswara ${ }^{131}$, D. Verkindt ${ }^{7}$, F. Vetrano ${ }^{57,58}$, A. Viceré ${ }^{57,58}$, S. Vinciguerra ${ }^{45}$, D. J. Vine ${ }^{50}$, J.-Y. Vinet ${ }^{53}$, S. Vitale ${ }^{10}$, T. Vo ${ }^{35}$, H. Vocca ${ }^{32,33}$, C. VorvicK ${ }^{37}$, D. Voss ${ }^{5}$, W. D. Vousden ${ }^{45}$, S. P. Vyatchanin ${ }^{49}$, A. R. Wade ${ }^{20}$,

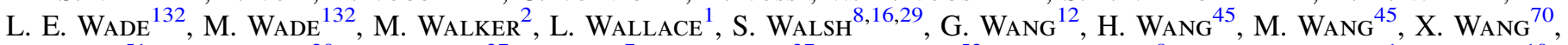
Y. WANG ${ }^{51}$, R. L. WARD ${ }^{20}$, J. WARNer ${ }^{37}$, M. WAs ${ }^{7}$, B. WeAver ${ }^{37}$, L.-W. WeI ${ }^{53}$, M. Weinert ${ }^{8}$, A. J. Weinstein ${ }^{1}$, R. Weiss ${ }^{10}$,

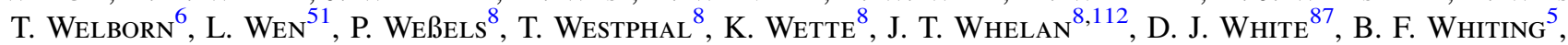
R. D. Williams ${ }^{1}$, A. R. Williamson ${ }^{92}$, J. L. Willis ${ }^{133}$, B. Willke" ${ }^{8,17}$, M. H. Wimmer ${ }^{8,17}$, W. Winkler ${ }^{8}$, C. C. Wipf ${ }^{1}$,

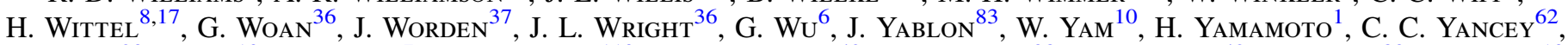

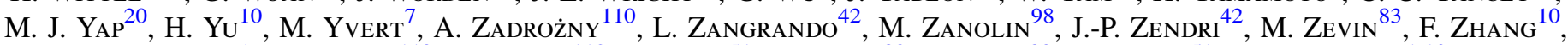

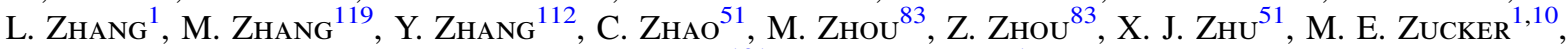

$$
\text { S. E. Zuraw }{ }^{101} \text {, AND J. Zweizig }{ }^{1}
$$

$\dagger$ Deceased, May 2015. $\ddagger$ Deceased, March 2015. (Ligo Scientific Collaboration and Virgo Collaboration)

\footnotetext{
${ }^{1}$ LIGO, California Institute of Technology, Pasadena, CA 91125, USA

${ }^{2}$ Louisiana State University, Baton Rouge, LA 70803, USA

${ }^{3}$ Università di Salerno, Fisciano, I-84084 Salerno, Italy

${ }^{4}$ INFN, Sezione di Napoli, Complesso Universitario di Monte S.Angelo, I-80126 Napoli, Italy

${ }^{5}$ University of Florida, Gainesville, FL 32611, USA

${ }^{6}$ LIGO Livingston Observatory, Livingston, LA 70754, USA

${ }^{7}$ Laboratoire d'Annecy-le-Vieux de Physique des Particules (LAPP), Université Savoie Mont Blanc, CNRS/IN2P3, F-74941 Annecy-le-Vieux, France

${ }^{8}$ Albert-Einstein-Institut, Max-Planck-Institut für Gravitationsphysik, D-30167 Hannover, Germany

${ }^{9}$ Nikhef, Science Park, 1098 XG Amsterdam, The Netherlands

${ }^{10}$ LIGO, Massachusetts Institute of Technology, Cambridge, MA 02139, USA

${ }^{11}$ Instituto Nacional de Pesquisas Espaciais, 12227-010 São José, dos Campos, SP, Brazil

${ }^{12}$ INFN, Gran Sasso Science Institute, I-67100 L'Aquila, Italy

${ }^{13}$ INFN, Sezione di Roma Tor Vergata, I-00133 Roma, Italy

${ }^{14}$ Inter-University Centre for Astronomy and Astrophysics, Pune 411007, India

${ }^{15}$ International Centre for Theoretical Sciences, Tata Institute of Fundamental Research, Bangalore 560012, India

${ }^{16}$ University of Wisconsin-Milwaukee, Milwaukee, WI 53201, USA

${ }^{7}$ Leibniz Universität Hannover, D-30167 Hannover, Germany

${ }^{18}$ Università di Pisa, I-56127 Pisa, Italy

${ }^{19}$ INFN, Sezione di Pisa, I-56127 Pisa, Italy

${ }^{20}$ Australian National University, Canberra, Australian Capital Territory 0200, Australia

${ }^{21}$ The University of Mississippi, Oxford, MS 38677, USA

${ }^{22}$ California State University Fullerton, Fullerton, CA 92831, USA

${ }^{23}$ LAL, Univ. Paris-Sud, CNRS/IN2P3, Université Paris-Saclay, Orsay, France

${ }^{24}$ Chennai Mathematical Institute, Chennai, India

${ }^{25}$ Università di Roma Tor Vergata, I-00133 Roma, Italy

${ }^{26}$ University of Southampton, Southampton SO17 1BJ, UK

${ }^{27}$ Universität Hamburg, D-22761 Hamburg, Germany

${ }^{28}$ INFN, Sezione di Roma, I-00185 Roma, Italy

${ }^{29}$ Albert-Einstein-Institut, Max-Planck-Institut für Gravitationsphysik, D-14476 Potsdam-Golm, Germany

${ }^{30}$ APC, AstroParticule et Cosmologie, Université Paris Diderot, CNRS/IN2P3, CEA/Irfu, Observatoire de Paris, Sorbonne Paris Cité, F-75205 Paris Cedex 13, France

${ }^{31}$ Montana State University, Bozeman, MT 59717, USA

${ }^{32}$ Università di Perugia, I-06123 Perugia, Italy

${ }^{33}$ INFN, Sezione di Perugia, I-06123 Perugia, Italy

${ }^{34}$ European Gravitational Observatory (EGO), I-56021 Cascina, Pisa, Italy

${ }^{35}$ Syracuse University, Syracuse, NY 13244, USA

${ }^{36}$ SUPA, University of Glasgow, Glasgow G12 8QQ, UK

${ }^{37}$ LIGO Hanford Observatory, Richland, WA 99352, USA

${ }^{38}$ Wigner RCP, RMKI, H-1121 Budapest, Konkoly Thege Miklós út 29-33, Hungary

${ }^{39}$ Columbia University, New York, NY 10027, USA

${ }^{40}$ Stanford University, Stanford, CA 94305, USA

${ }^{41}$ Università di Padova, Dipartimento di Fisica e Astronomia, I-35131 Padova, Italy

${ }^{42}$ INFN, Sezione di Padova, I-35131 Padova, Italy

${ }^{43}$ CAMK-PAN, 00-716 Warsaw, Poland

${ }^{44}$ Astronomical Observatory Warsaw University, 00-478 Warsaw, Poland

${ }_{45}^{45}$ University of Birmingham, Birmingham B15 2TT, UK

${ }^{46}$ Università degli Studi di Genova, I-16146 Genova, Italy

${ }^{47}$ INFN, Sezione di Genova, I-16146 Genova, Italy

${ }^{48}$ RRCAT, Indore MP 452013, India

${ }^{49}$ Faculty of Physics, Lomonosov Moscow State University, Moscow 119991, Russia

${ }^{50}$ SUPA, University of the West of Scotland, Paisley PA1 2BE, UK

${ }^{51}$ University of Western Australia, Crawley, Western Australia 6009, Australia

${ }^{52}$ Department of Astrophysics/IMAPP, Radboud University Nijmegen, P.O. Box 9010, 6500 GL Nijmegen, The Netherlands

${ }_{53}$ Artemis, Université Côte d'Azur, CNRS, Observatoire Côte d'Azur, CS F-34229, Nice cedex 4, France

${ }^{54}$ MTA Eötvös University, "Lendulet" Astrophysics Research Group, Budapest 1117, Hungary
} 


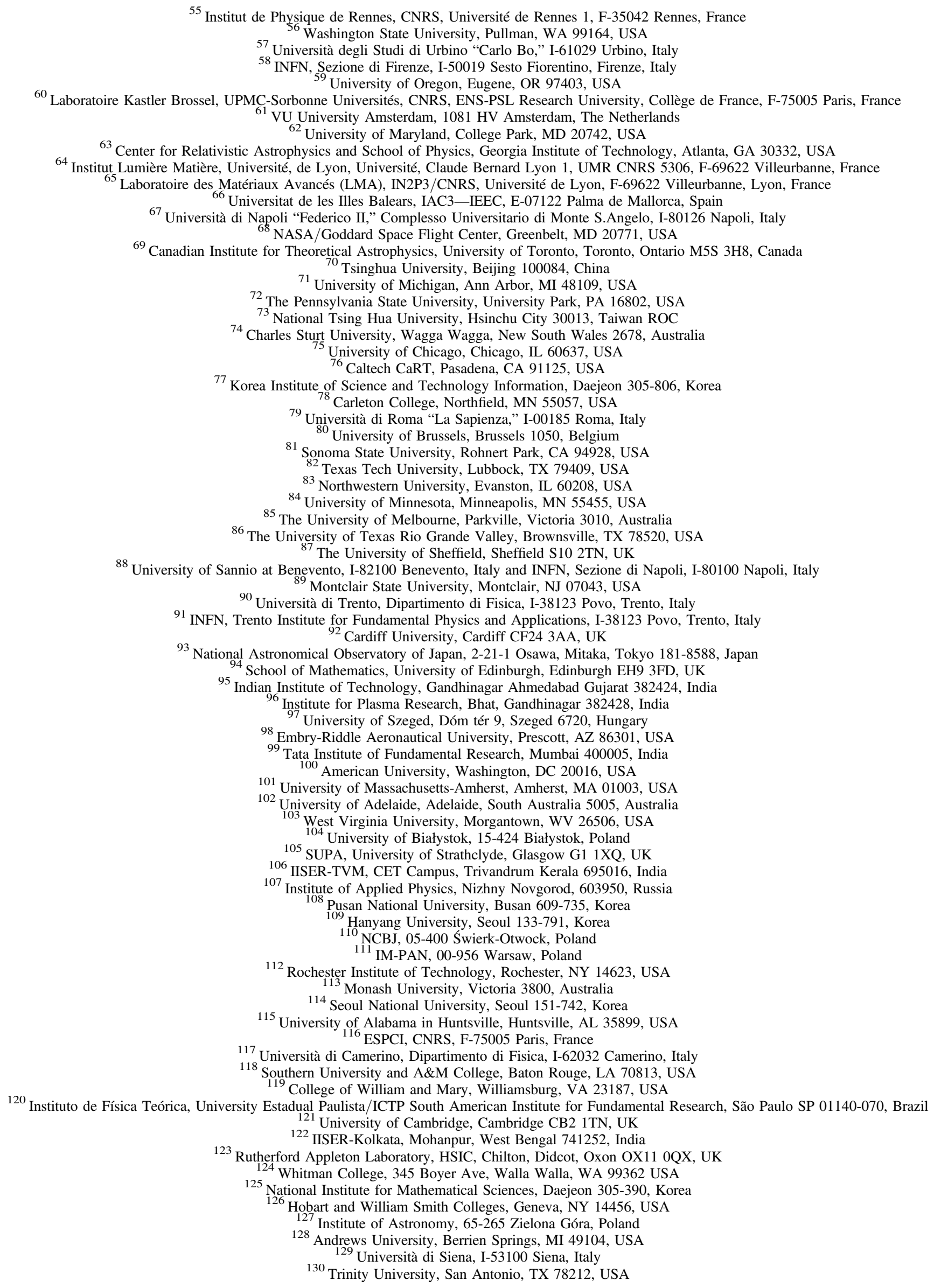




\author{
${ }^{131}$ University of Washington, Seattle, WA 98195 , USA \\ ${ }_{132}$ Kenyon College, Gambier, OH 43022, USA \\ ${ }_{133}$ Abilene Christian University, Abilene, TX 79699, USA \\ Received 2016 January 29; accepted 2016 February 3; published 2016 February 11
}

\begin{abstract}
The discovery of the gravitational-wave (GW) source GW150914 with the Advanced LIGO detectors provides the first observational evidence for the existence of binary black hole $(\mathrm{BH})$ systems that inspiral and merge within the age of the universe. Such BH mergers have been predicted in two main types of formation models, involving isolated binaries in galactic fields or dynamical interactions in young and old dense stellar environments. The measured masses robustly demonstrate that relatively "heavy" BHs $\left(\gtrsim 25 M_{\odot}\right)$ can form in nature. This discovery implies relatively weak massive-star winds and thus the formation of GW150914 in an environment with a metallicity lower than about $1 / 2$ of the solar value. The rate of binary-BH $(\mathrm{BBH})$ mergers inferred from the observation of GW150914 is consistent with the higher end of rate predictions $\left(\gtrsim 1 \mathrm{Gpc}^{-3} \mathrm{yr}^{-1}\right)$ from both types of formation models. The low measured redshift $(z \simeq 0.1)$ of GW150914 and the low inferred metallicity of the stellar progenitor imply either BBH formation in a low-mass galaxy in the local universe and a prompt merger, or formation at high redshift with a time delay between formation and merger of several Gyr. This discovery motivates further studies of binary-BH formation astrophysics. It also has implications for future detections and studies by Advanced LIGO and Advanced Virgo, and GW detectors in space.
\end{abstract}

Key words: gravitational waves - stars: black holes - stars: massive

\section{INTRODUCTION}

When in the 1970s the mass of the compact object in the X-ray binary (XRB) Cygnus X-1 was measured to exceed the maximum mass of a neutron star (Webster \& Murdin 1972; Bolton 1972), black holes (BHs) turned from a theoretical concept into an observational reality. Around the same time and over several years, evidence for supermassive BHs in the centers of galaxies mounted (see Kormendy \& Richstone 1995). The formation of the stellarmass BHs found in XRBs is associated with the core collapse (and potential supernova $[\mathrm{SN}]$ explosion) of massive stars when they have exhausted their nuclear fuel (e.g., Heger et al. 2003). The origin of supermassive BHs is less clear. They may have small seeds that originate from "heavy" stellar-mass BHs (more massive than about $25 M_{\odot}$ ) or large seeds from intermediate-mass $\mathrm{BHs}$ formed in the earliest generations of massive stars or directly from large clouds (see Volonteri 2010).

The gravitational-wave (GW) signal GW150914 detected on 2015 September 14 09:50:45 UTC by the Advanced LIGO (aLIGO) detectors (Abbott et al. 2016a, hereafter LVC16a; henceforth we refer similarly to all other forthcoming papers from the LIGO-Virgo Collaborations and related to GW150914) has been shown to originate from the coalescence of a binary BH (BBH) with masses of $36_{-4}^{+5} M_{\odot}$ and $29_{-4}^{+4} M_{\odot}$ (in the source frame, see Section 2). This GW discovery provides the first robust confirmation of several theoretical predictions: (i) that "heavy" BHs exist, (ii) that BBHs form in nature, and (iii) that BBHs merge within the age of the universe at a detectable rate.

The inspiral and merger of binaries with BHs or neutron stars (NSs) have been discussed as the primary source for groundbased GW interferometers for many decades (e.g., Thorne 1987; Schutz 1989). The existence of GWs was established with radio observations of the orbital decay of the first binary pulsar, PSR B1913+16 (Hulse \& Taylor 1975; Taylor \& Weisberg 1982). Even before the binary pulsar discovery, Tutukov \& Yungelson (1973) described the evolution of isolated massive binaries (i.e., those not influenced dynamically by surrounding stars) and predicted the formation of binary compact objects, including BBHs. Some of the first population studies of massive stellar binaries and their evolution even predicted that BBH mergers could dominate detection rates for ground-based GW interferometric detectors (Lipunov et al. 1997). Furthermore, Sigurdsson \& Hernquist (1993) recognized that dense star clusters provide another possible way of forming merging BBHs: BHs in dense star clusters quickly become the most massive objects, sink toward the cluster core, subsequently form pairs through dynamical interactions, and are most commonly ejected in binary configurations with inspiral times shorter than the age of the universe. For the most recent review articles on the formation of binary compact objects in galactic fields and dense stellar systems, see Postnov \& Yungelson (2014) and Benacquista \& Downing (2013), respectively.

In this paper we discuss GW150914 in the context of astrophysical predictions in the literature and we identify the most robust constraints on BBH formation models. In Section 2 we report the properties of GW150914. In Section 3 and Section 4 we discuss the implications of the measured $\mathrm{BH}$ masses and distance to the source. In Section 5 and Section 6 we examine conclusions that can be drawn from the GW constraints on the orbital eccentricity, $\mathrm{BH}$ spins, and BBH merger rates. In Section 7 we discuss prospects for future detections and the types of astrophysical studies we would need to further advance our understanding of $\mathrm{BBH}$ formation. In Section 8 we summarize our key conclusions.

\section{THE PROPERTIES OF GW150914}

GW150914 was discovered first through a low-latency search for GW transients, and later in subsequent match-filter analyses of 16 days of coincident data collected by the two aLIGO detectors between September 12 and October 20 (LVC16a). The signal matches the waveform expected for the inspiral, merger, and ringdown from a compact binary. In $0.2 \mathrm{~s}$ it swept in frequency from 35 to $250 \mathrm{~Hz}$, reaching a peak GW strain of $1.0 \times 10^{-21}$ with a signal-to-noise ratio of 24 (LVC16b, LVC16c).

Consideration of these basic signal properties of frequency and frequency derivative indicate that the source is a $\mathrm{BH}$ merger (LVC16a). Coherent Bayesian analyses (LVC16d) using advanced waveforms (Hannam et al. 2014; Pürrer 2014; Taracchini et al. 2014; Khan et al. 2015) allow us to 
measure several of the source physical parameters (all quoted at $90 \%$ credible level). In the detector frame, the chirp mass ${ }^{1}$ is $30_{-2}^{+2} M_{\odot}$ and the total mass is $71_{-4}^{+5} M_{\odot}$; the mass ratio is $0.82_{-0.21}^{+0.16}$ and the luminosity distance is determined to be $410_{-180}^{+160} \mathrm{Mpc}$ (redshift $0.09_{-0.04}^{+0.03}$ ). The two BH masses in the source frame then are $36_{-4}^{+5} M_{\odot}$ and $29_{-4}^{+4} M_{\odot}$, and the chirp mass in the source frame is $28_{-2}^{+2} M_{\odot}$. The source-frame mass and spin of the final $\mathrm{BH}$ are $62_{-4}^{+4} M_{\odot}$ and $0.67_{-0.07}^{+0.05}$ and the source is localized to a sky area of $600 \mathrm{deg}^{2}$ (see also LVC16d; LVC16h). The signal does not show deviations from the expectations of general relativity, as discussed in detail in LVC16e.

Assuming that the source-frame BBH merger rate is constant within the volume in which GW150914 could have been detected (found to extend out to redshift of $\simeq 0.5$ ) and that GW150914 is representative of the underlying BBH population, the BBH merger rate is inferred to be $2-53 \mathrm{Gpc}^{-3} \mathrm{yr}^{-1}$ in the comoving frame at the $90 \%$ credible level (Kim et al. 2003; LVC16f). The match-filter searches of these 16 days of coincident data also revealed a number of sub-threshold triggers with associated probabilities of being astrophysical or noise in nature (LVC16b). If we account for the probability of these sub-threshold triggers and we consider a wide range of models for the underlying $\mathrm{BBH}$ mass distribution, the estimated $\mathrm{BBH}$ merger rates extend to the range 2 $-400 \mathrm{Gpc}^{-3} \mathrm{yr}^{-1}$ (Farr et al. 2015; LVC16f).

\section{BH MASSES IN MERGING BINARIES}

\subsection{Brief Review of Measured BH Masses}

Prior to the discovery of GW150914, our knowledge of stellar BH masses has come from the study of XRBs where a compact object accretes matter from a stellar companion (e.g., McClintock \& Remillard 2006). Dynamical compact-object mass measurements in these binaries rely on measurements of the system's orbital period, the amplitude of the stellarcompanion's radial velocity curve, and quantitative constraints on the binary inclination and the companion mass (e.g., Casares $\&$ Jonker 2014). When the mass of the compact object is found to exceed $3 M_{\odot}$, which is the conservative upper limit for stable NSs (Rhoades \& Ruffini 1974; Kalogera \& Baym 1996), then the XRB is considered to host a BH. At present, $22 \mathrm{BH}$ XRBs have confirmed dynamical mass measurements, 19 of which lie in our Galaxy. For the majority of the systems, the measured $\mathrm{BH}$ masses are $5-10 M_{\odot}$, while some have masses of $10-20 M_{\odot}{ }^{2} \mathrm{BH}$ masses have been claimed to be measured dynamically in two other extragalactic systems, IC10 X-1 (Prestwich et al. 2007; Silverman \& Filippenko 2008, $M_{\mathrm{BH}}=$ 21-35 $M_{\odot}$ ) and NGC300 X-1 (Crowther et al. 2010, $M_{\mathrm{BH}}=$ 12-24 $\left.M_{\odot}\right)$. On the basis of these observations, Bulik et al. (2011) argue that these two systems are likely immediate progenitors of BBH systems. However, recent work casts doubt on these BH masses: it now appears more likely that the measured velocities are due to stellar-wind features instead of the BH companion's orbital motion (Laycock et al. 2015, and references therein), and therefore we do not consider the claimed BH masses in these systems as reliable.

\footnotetext{
1 The chirp mass is $\mathcal{M}=\left(m_{1} m_{2}\right)^{3 / 5} /\left(m_{1}+m_{2}\right)^{1 / 5}$, where $m_{1}$ and $m_{2}$ are the component masses.

2 For probability distribution functions of measured $\mathrm{BH}$ masses see Farr et al. (2011) and Özel et al. (2010).
}

All of these observed BH systems are found in low stellar density galactic fields. Based on multi-wavelength electromagnetic studies of X-ray point sources, BH XRBs have also been claimed to exist in globular clusters (Maccarone et al. 2007; Chomiuk et al. 2013, and references therein); however, dynamical mass measurements for these systems have not been possible, and hence reliable $\mathrm{BH}$ mass constraints are not available.

Both BHs of the GW150914 coalescence are more massive than the BHs in known XRBs with reliably measured masses: this $G W$ discovery provides the most robust evidence for the existence of "heavy" $\left(\gtrsim 25 M_{\odot}\right)$ stellar-mass BHs. In what follows, we review our current understanding of $\mathrm{BH}$ and $\mathrm{BBH}$ formation both in isolation and in dense environments, and we examine the implications of the high GW150914 BH masses.

\subsection{Predicted Masses for Single BHs}

$\mathrm{BHs}$ are expected to form at the end of the nuclear lifetimes of massive stars. The stellar core collapses to form a proto-NS and the occurrence and strength of an SN explosion determines how much material is ejected, and whether a BH is formed. Fryer \& Kalogera (2001) distinguish BH formation through partial or full fallback of the initially exploding envelope, or through the complete collapse of the $\mathrm{BH}$ progenitor due to a core collapse that is not powerful enough to drive an explosion. Fryer et al. (2012) find that the transition from NS to BH formation occurs at initial progenitor masses of $\simeq 18-20 M_{\odot}$ and the transition from fallback to complete $\mathrm{BH}$ collapse takes place at initial progenitor masses of $\simeq 40 M_{\odot}$. Other studies (e.g., Ugliano et al. 2012) find that either the SN is successful and an NS is formed, or the whole star collapses to a BH; there is a range of progenitor masses $\left(15-40 M_{\odot}\right.$ for solar metallicity) where either an NS or a BH could form.

This relatively simple picture of $\mathrm{BH}$ formation from single stars is greatly affected by several key factors: the strength of massive-star winds and their dependence on the star's metallicity (Z, e.g., Maeder 1992), stellar rotation (e.g., de Mink et al. 2009), and the microphysics of stellar evolution. These factors affect the relationship between the initial progenitor mass and the stellar (core) mass at the time of collapse, and thus the mass of the resulting $\mathrm{BH}$.

Winds are understood to be radiation-driven. Their strengths depend on stellar properties, but cannot be derived from first principles; instead they are empirically derived and calibrated based on observations (for a review, see Smith 2014). Over the last decades, wind strengths for different evolutionary stages have been significantly revised, mainly downwards, leading to more massive progenitors at core collapse (for a review, see Vink 2008). In general, stars at lower metallicities exhibit weaker winds, since the lower metal content reduces opacity, enables easier radiation transport, and reduces radiation momentum transfer and hence mass loss from the stellar surface. The functional dependence on metallicity is also empirically constrained by studying massive stars in environments of different metallicities. However, the range in metallicities probed by observations is much smaller than the range where massive stars are formed over cosmic history, and hence extrapolations to metallicities orders of magnitude smaller than solar $Z_{\odot}$ (i.e., $Z_{\odot}=0.02$ ) are adopted. Although we have no way of validating such extrapolations, here we consider the published low-metallicity models.

Heger et al. (2003) and Mapelli et al. (2009) were among the first to examine how compact object formation depends on progenitor masses, stellar winds, and metallicity, albeit in a rather 

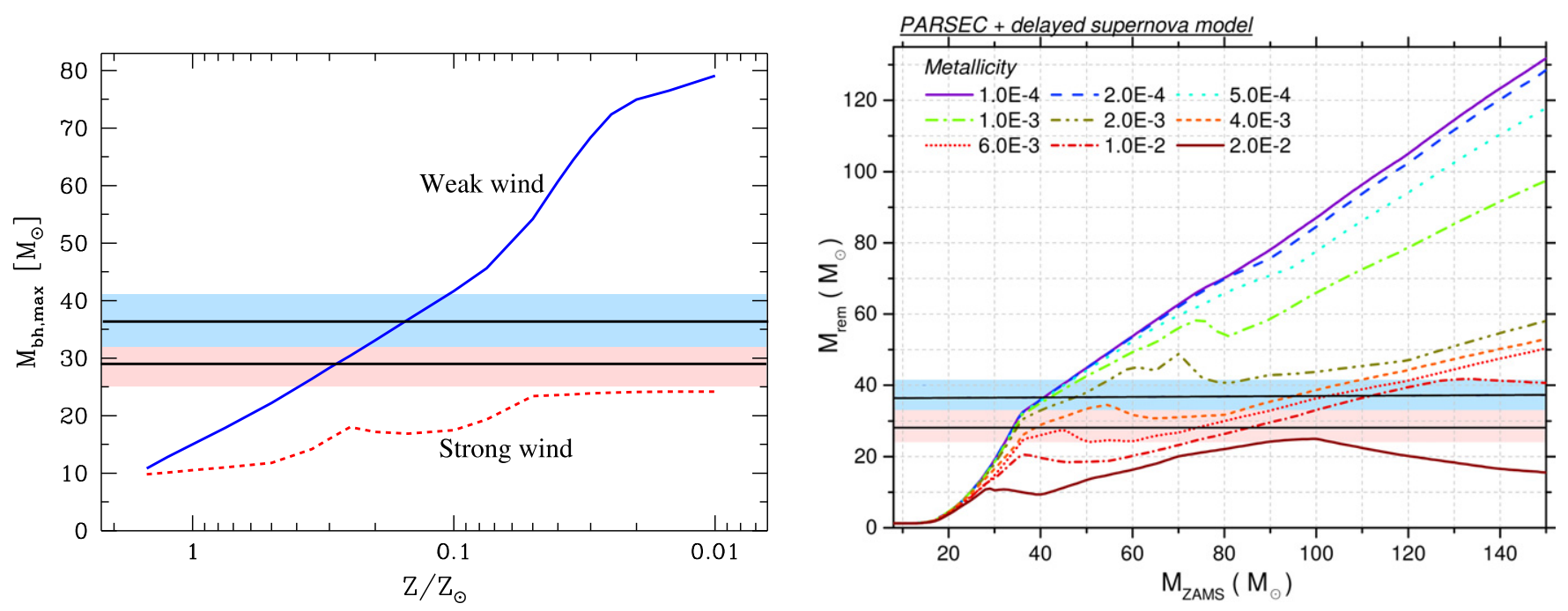

Figure 1. Left: dependence of maximum BH mass on metallicity $Z$, with $Z_{\odot}=0.02$ for the old (strong) and new (weak) massive-star winds (Figure 3 from Belczynski et al. 2010a). Right: compact-remnant mass as a function of zero-age main-sequence (ZAMS; i.e., initial) progenitor mass for a set of different (absolute) metallicity values (Figure 6 from Spera et al. 2015). The masses for GW150914 are indicated by the horizontal bands.

qualitative framework. Quantitatively, Belczynski et al. (2010a) and later Mapelli et al. (2013) and Spera et al. (2015) showed that adopting the latest wind prescriptions (Vink 2008) significantly increases the stellar mass at core collapse and thus the maximum $\mathrm{BH}$ mass that can form from single stars, although the exact relation between initial mass and final $\mathrm{BH}$ mass depends on the details of the wind prescription (see Figure 1).

Stellar rotation can lead to angular momentum transport and extra mixing in the stellar interiors. In extreme cases, the evolution of the star can be significantly altered, avoiding expansion of the star into a giant (Maeder 1987). It has been proposed that rapid rotation, especially at low metallicities, where winds and associated angular momentum losses are weaker, or in close binaries, where tides may replenish the angular momentum, may play a significant role in the formation of more massive BHs (de Mink et al. 2009; Mandel $\&$ de Mink 2016; Marchant et al. 2016). Nevertheless, there are no calculations that find $\mathrm{BHs}$ more massive than $30 M_{\odot}$ unless the metallicity is lower than $Z_{\odot}$.

Stellar properties at core collapse and the ensuing compactremnant masses have also been shown to depend, albeit much more weakly, on the treatment of microphysics in stellar structure and evolution codes, especially on assumptions regarding convective overshooting and resultant mixing (Jones et al. 2015). Finally, Fryer et al. (2012) and Spera et al. (2015) investigate how basic properties of the SN explosion might affect remnant masses at different metallicities. They show that remnant masses in excess of $\simeq 12 M_{\odot}$ at $Z_{\odot} \quad\left(\simeq 30 M_{\odot}\right.$ at $\left.1 / 100 Z_{\odot}\right)$ are formed through complete collapse of their progenitors. Therefore, the masses of BHs in "heavy" BBH mergers only carry information about the evolution leading up to the collapse and not about the SN mechanism.

The measured masses of the merging BHs in GW150914 show that stellar-mass BHs as massive as $32 M_{\odot}$ (the lower limit on the more massive $\mathrm{BH}$ at $90 \%$ credible level) can form in nature. Given our current understanding of BH formation from massive stars, using the latest stellar wind, rotation, and metallicity models, we conclude that the GW150914 BBH most likely formed in a low-metallicity environment: below $\simeq 1 / 2 Z_{\odot}$ and possibly below $\simeq 1 / 4 Z_{\odot}$ (Belczynski et al. 2010a; Mapelli et al. 2013; Spera et al. 2015).
It is, in principle, possible that "heavy" BHs are formed through indirect paths that do not require a low metallicity, but we consider this very unlikely. For example, the formation of "heavy" BHs through the dynamical mergers of lower-mass BHs with massive stars in young clusters has been considered. However, these models adopt the optimistic assumption that in such mergers, even for grazing collisions, all of the mass is retained, leading to significant BH mass growth (Mapelli \& Zampieri 2014; Ziosi et al. 2014). Stellar collisions in dense stellar environments (see Portegies Zwart et al. 1999) could potentially produce stars massive enough to form "heavy" BHs, but these objects are also subject to strong winds and intense mass loss unless they are stars of low metallicity (e.g., Glebbeek et al. 2009). Finally, formation of "heavy" BHs from the mergers of lower-mass BHs in clusters is unlikely because most dynamically formed merging $\mathrm{BBHs}$ are ejected from the host cluster before merger (Rodriguez et al. 2015, see their Figure 2).

\subsection{BBH Masses from Isolated Binary Systems}

The fact that the majority of massive stars are members of binary systems with a roughly flat mass-ratio distribution (Kobulnicky \& Fryer 2007; Sana et al. 2012; Kobulnicky et al. 2014) provides the opportunity for BBH formation in isolated binary systems. In that case, the masses of BHs depend not only on the initial mass of the star and metallicity, but also on any binary interactions. The development of binary population models focused on the formation of double compact objects goes back to Kornilov \& Lipunov (1983) and Dewey \& Cordes (1987), but the first population models to account for BBH formation appeared a decade later starting with Tutukov \& Yungelson (1993). Several groups have explored different aspects of $\mathrm{BBH}$ formation from isolated binaries at varying levels of detail (many reviewed by Kalogera et al. 2007; Vanbeveren 2009; Postnov \& Yungelson 2014). Models find that $\mathrm{BBH}$ formation typically progresses through the following steps: (i) stable mass transfer between two massive stars, although potentially non-conservative (i.e., with mass and angular momentum losses from the binary), (ii) the first core collapse and $\mathrm{BH}$ formation event, (iii) a second mass transfer 
phase that is dynamically unstable leading to inspiral in a common envelope (in which the first $\mathrm{BH}$ potentially grows slightly in mass; O'Shaughnessy et al. 2005a), (iv) the second core-collapse event leading to BBH formation, and (v) inspiral due to GW emission and merger. Dominik et al. (2012) found that the vast majority of BBH mergers follow this evolutionary path: $99 \%$ at solar metallicity and $90 \%$ at $0.1 Z_{\odot}$. Alternative formation pathways, avoiding mass transfer and common envelope, may be possible if massive stars remain rapidly rotating, stay chemically homogeneous through their lifetimes, remain compact, and do not become giant stars (see de Mink et al. 2009; Mandel \& de Mink 2016; Marchant et al. 2016).

Most studies indicate that model predictions, in particular merger rates, but also probability distributions of $\mathrm{BBH}$ properties, are affected by a considerable number of physical factors and associated parameters, albeit at different levels of sensitivity: (i) initial binary properties (masses, mass ratios, and orbital periods), (ii) stellar evolution models including metallicity-dependent winddriven mass loss, (iii) mass and associated angular momentum transfer between binary components and loss from the systems, (iv) treatment of tidal evolution, (v) treatment of commonenvelope evolution, and (vi) BH natal kicks. The significance of (v) and (vi) has been discussed recently for the StarTrack (Belczynski et al. 2008a) models by Dominik et al. (2012) and Belczynski et al. (2015). Recently, de Mink \& Belczynski (2015) concluded that the current uncertainties in initial binary properties (i) do not dramatically change the rates. The other factors, i.e., (ii)-(vi), have been consistently identified as important, not just for rate predictions, but also for predictions of $\mathrm{BH}$ mass spectra in merging BBHs.

As we have discussed, the GW150914 masses favor the newer, weaker stellar winds and metallicities below $Z_{\odot}$. Quantitative models for $\mathrm{BH}$ and $\mathrm{BBH}$ formation considering such conditions have appeared only in the past five years, starting with Belczynski et al. (2010b), and in numerous follow-up studies (Dominik et al. 2012, 2013, 2015; Belczynski et al. 2015; Spera et al. 2015). Dominik et al. (2013) fold in cosmological effects, accounting for redshift evolution of the formation rate and metallicity (down to $Z=10^{-4}$ ). With the extension to such low metallicities, the strong dependence on the common-envelope treatment found earlier (Dominik et al. 2012) is weakened in the case of formation of BHs more massive than $20 M_{\odot}$. In fact, it is striking that, once full metallicity evolution is included, $\mathrm{BBH}$ systems that merge within the age of the universe and have total masses as high as $\sim 100 M_{\odot}$ are rather generically formed regardless of other model assumptions; still, predicted detectable samples seem to be dominated by less massive BBH systems (Belczynski et al. 2014; Dominik et al. 2015).

On the extreme low-metallicity end, it has been proposed that $\mathrm{BBH}$ formation is also possible in the case of stellar binaries at zero metallicity (Population III [PopIII] stars; see Belczynski et al. 2004; Kinugawa et al. 2014). The predictions from these studies are even more uncertain, since we have no observational constraints on the properties of first-generation stellar binaries (e.g., mass function, mass ratios, orbital separations). However, if one assumes that the properties of PopIII massive binaries are not very different from binary populations in the local universe (admittedly a considerable extrapolation), then recently predicted $\mathrm{BBH}$ total masses agree astonishingly well with GW150914 and can have sufficiently long merger times to occur in the nearby universe (Kinugawa et al. 2014). This is in contrast to the predicted mass properties of low (as opposed to zero) metallicity populations, which show broader distributions (Belczynski et al. 2015).

We conclude that predictions from a broad range of models for $B B H$ formation from isolated binaries are consistent with the GW150914 masses provided that newer, weaker massivestar winds and extrapolations to metallicities of $1 / 2 Z_{\odot}$ or lower are adopted. More calculations of massive binary evolution with updated wind prescriptions and taking cosmological evolution into account are needed to fully exploit the new information that would be provided by additional $\mathrm{GW}$ detections.

\subsection{BBH Masses from Dense Stellar Environments}

Over the last few decades our understanding of the evolution of BHs in dense stellar clusters has evolved considerably. Based on early analyses (Kulkarni et al. 1993; Sigurdsson \& Hernquist 1993) BHs form in clusters from massive stars and quickly mass segregate to the center through dynamical friction (on a timescale shorter than the overall relaxation time by a factor that is the ratio of the mass of the typical $\mathrm{BH}$ to the mass of an average background star). In these high-density conditions, BHs dynamically interact, forming binaries, and often are ejected from the cluster. Such dynamical interactions preferentially keep the heaviest objects in binaries and eject the lightest, producing heavier binaries and driving mass ratios closer to unity (Heggie 1975). Portegies Zwart \& McMillan (2000) presented the first significant $N$-body simulation of equal-mass BHs in a dense cluster, and they found that the ejected $\mathrm{BBH}$ systems are sufficiently eccentric that they will merge within the age of the universe at a rate important for LIGO/Virgo observations. Since then, studies of varying levels of detail have examined $\mathrm{BBH}$ formation in clusters and have identified the importance of three-body interactions for hardening binaries to the point they can merge in a Hubble time, pointing out that these interactions are also responsible for dynamical ejections (Gültekin et al. 2004, 2006; Kocsis et al. 2006; Banerjee et al. 2010; Bae et al. 2014) as well as in galactic centers (Miller \& Lauburg 2009; O'Leary et al. 2009; Kocsis \& Levin 2012; Tsang 2013). GW kicks (Zlochower \& Lousto 2015 and references therein) can also eject post-merger, single BHs from their host clusters. Throughout these studies BHs are assumed to be of a single fixed mass (typically $10 M_{\odot}$ ). Therefore, although their results are relevant for understanding the effects of stellar dynamics on $\mathrm{BBH}$ formation and evolution and the expected merger rates (Section 6), they cannot be used to determine the expected masses of $\mathrm{BBH}$ mergers formed in dynamical environments.

O'Leary et al. (2006, 2007) and Sadowski et al. (2008) presented the first $\mathrm{BBH}$ population predictions from dense clusters with a $\mathrm{BH}$ mass spectrum. Their treatment of the effects of stellar dynamics was based on simple cross sections and a static density background. Nevertheless, their results generically produced $\mathrm{BBH}$ mergers in the local universe with $\mathrm{BH}$ masses of several tens of solar masses.

The first simulations to account in detail for both binary evolution and stellar dynamics with a $\mathrm{BH}$ mass spectrum and with realistic numbers of particles were by Downing et al. (2010, 2011) and by Morscher et al. (2013, 2015). Morscher et al. (2015) and Rodriguez et al. (2016) further accounted for a population of globular clusters with varying cluster properties (mass, density, and metallicity). Examination of these results indicates, very much like the models of isolated binary 


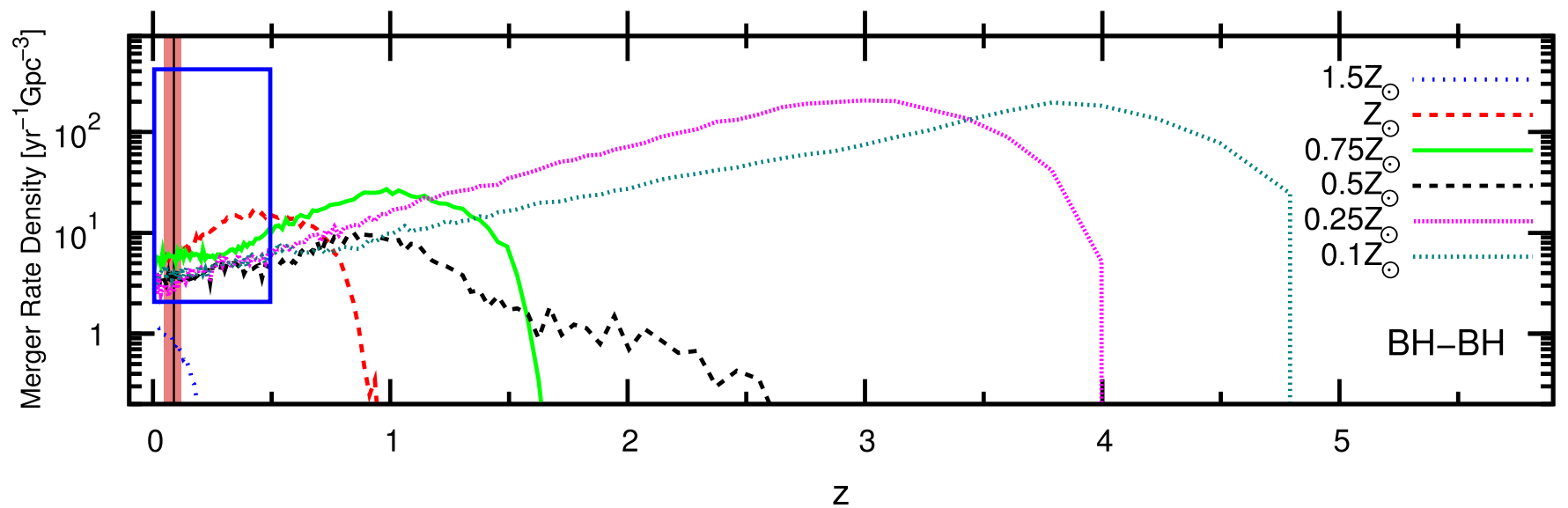

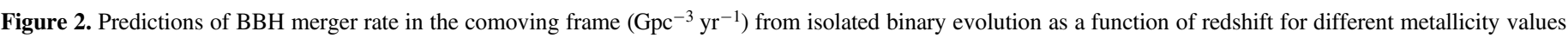

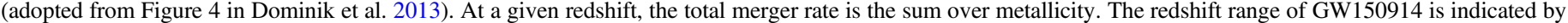

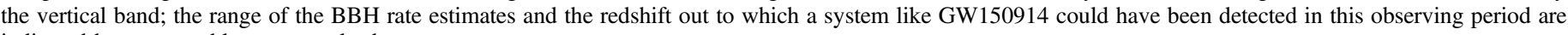
indicated by an open blue rectangular box.

evolution, that clusters of lower metallicities produce $\mathrm{BBH}$ mergers of higher masses, with chirp masses in excess of $10 M_{\odot}$ and up to $25-30 M_{\odot}$ (the chirp mass of GW150914 is $\left.28_{-2}^{+2} M_{\odot}\right)$. We note that none of these studies incorporate the newer, weaker winds leading to more massive BHs adopted by some of the models for isolated binaries (Section 3.3). Such a modification applied to clusters will unavoidably increase the $\mathrm{BBH}$ masses from clusters even further. BBH populations are also predicted to form in young, open clusters (Goswami et al. 2014; Ziosi et al. 2014) with "heavy" masses. In this case, BBHs are formed mostly through dynamical exchanges in three-body encounters of single $\mathrm{BHs}$ with binaries containing one or two BHs.

We conclude that BBH formation in dense star clusters is consistent with GW150914, provided that the clusters have typical metallicities lower than $Z_{\odot}$ in order to form sufficiently massive merging BBHs. Most of these mergers occur outside the clusters following dynamical BBH ejection. Although, under optimistic assumptions, formation of "heavy" BHs at solar metallicity has been discussed through stellar or BH-star mergers in young clusters, such paths require chains of dynamical interactions for eventual $\mathrm{BBH}$ formation. Cluster models with more detailed treatment of binary evolution, dynamics, updated stellar winds, and exploration of cluster properties are needed to determine the robustness of the predicted BBH mass spectra.

\section{BBH MERGERS IN THE NEARBY UNIVERSE}

Apart from the BH masses of the binary system, another important measurement of GW150914 is its luminosity distance in the range of $230-570 \mathrm{Mpc}$ (at $90 \%$ credible level) which corresponds to a redshift of $0.05-0.12$ and an age of the universe of $\simeq 12.2-13.1 \mathrm{Gyr}$ at the time of the merger (using Planck Collaboration et al. 2015). The specific implications of this measurement vary, depending on whether GW150914 originated from isolated binary evolution or from dynamical interactions in a dense stellar environment.

In the case of dynamical origin, mergers of such "heavy" BBHs in the local universe fit comfortably. Models of dynamical BBH formation (Portegies Zwart \& McMillan 2000; Miller \& Lauburg 2009; O'Leary et al. 2009; Downing et al. 2011; Kocsis \& Levin 2012; Tsang 2013; Ziosi et al.
2014; Morscher et al. 2015; Rodriguez et al. 2015, 2016) show that stellar and $\mathrm{BH}$ interactions take about $\sim 1 \mathrm{Gyr}$ to form $\mathrm{BBHs}$, which have a wide range of delay times between formation and merger for BBHs from old and young clusters.

In the case of a $\mathrm{BBH}$ merger from an isolated binary at a low metallicity, there is a continuum of possibilities in between two extremes: the BBH progenitor of GW150914 could have formed in the local universe with a short merger delay time, or it could have formed at a higher redshift with a long merger delay time. We cannot distinguish between these two extremes with the observation of this single event.

Short merger times are typically favored. One of the most recent isolated binary model predictions (Dominik et al. 2013) shows preference for merger times of $\simeq 10-300$ Myr. However, low-metallicity star formation is rare in nearby galaxies. The age and metallicity distribution of a large sample of nearby galaxies from the Sloan Digital Sky Survey with a median redshift of 0.13 shows that very few galaxies have low metallicities, all of which are low-mass $\left(<10^{9} M_{\odot}\right)$ and have relatively young stellar populations $(<1 \mathrm{Gyr}$, Gallazzi et al. $2005)$. In any case, the well measured mass-metallicity relation (Tremonti et al. 2004) implies that BBH formation paths with merger delay times below $\sim 1 \mathrm{Gyr}$ require that the source originated in nearby $(z<0.2)$ low-mass, young galaxies.

Alternatively, the $\mathrm{BBH}$ system may have formed much earlier (e.g., $z \gtrsim 2$ ), when low-metallicity star formation was more common (see Madau \& Dickinson 2014), but then it must have taken much longer to merge ( 5-10 Gyr). Such long merger delay times are often disfavored significantly compared to short delays (by factors of $10-100$; see, e.g., Dominik et al. 2013).

To present a more quantitative discussion, we consider the study by Dominik et al. (2013) in more detail. They discuss $\mathrm{BBH}$ formation from isolated binaries, accounting for the dependence on star formation, galaxy-mass, and metallicity evolution from the local universe to cosmological redshifts and find that most local BBH mergers originate from star formation in the first few Gyr of the universe and with long merger delay times (see also Dominik et al. 2015). Figure 2 (adopted from Figure 4 of Dominik et al. 2013) indicates that the BBH merger rate of binaries with metallicities of $1 / 2,1 / 4$, and $1 / 10 Z_{\odot}$ increases with redshift and peaks at redshifts of 1,3 , and 4 , 


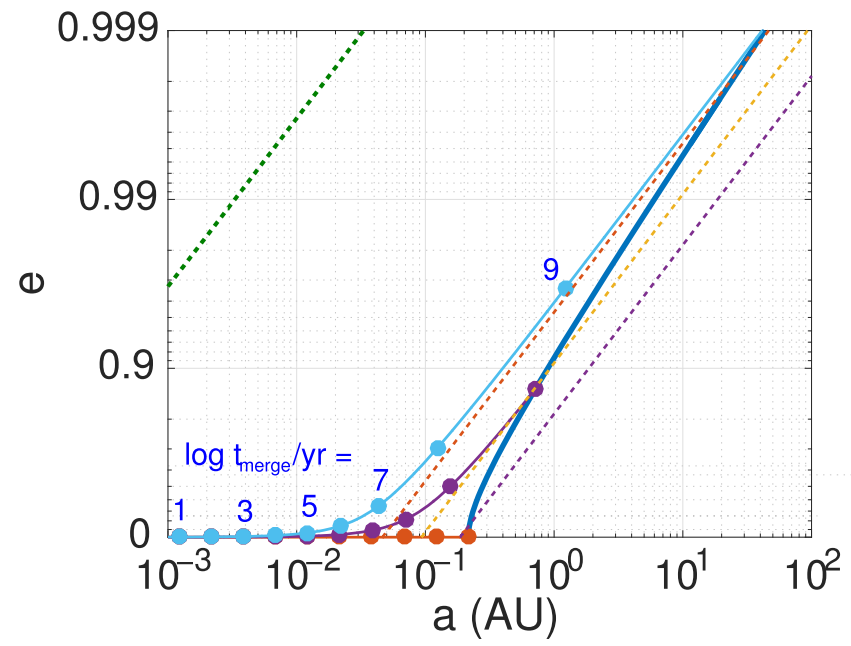

Figure 3. Allowed initial BBH semimajor axis and eccentricity in order to merge within $10 \mathrm{Gyr}$ (left of the thick solid blue line) for a $\mathrm{BBH}$ with the GW150914 masses. The thin solid lines with circles represent the evolutionary trajectories of individual example systems, starting at the edge of the allowed range (the circles give the time to merger of $\log \left(t \mathrm{yr}^{-1}\right)=1,2,3,4, \ldots 10$, from left to right). The dashed lines denote periastron separations of 10,20 , and $40 R_{\odot}$ (left to right: orange, yellow, purple). The green dotted line shows the trajectory of a binary that has a remaining eccentricity of 0.1 at a GW frequency of $10 \mathrm{~Hz}$.

respectively, i.e., at distances much larger than the measured GW150914 luminosity distance. The local $(z \sim 0.1) \mathrm{BBH}$ merger rates at such low metallicities are suppressed by factors of 10-100 compared to higher redshifts, but they are still comparable (within a factor of about 2) to the high-metallicity local merger rate densities that produce lower-mass BBHs.

To further study the potential progenitors of GW150914 and their expected merger time, we plot in Figure 3 the allowed parameter range for the initial (right after $\mathrm{BBH}$ formation) semimajor axis $(a)$ and eccentricity $(e)$ of the $\mathrm{BBH}$ orbit that produces a merger within $10 \mathrm{Gyr}$, using the point-mass approximation of Peters (1964). Binaries with long delay times originate close to the thick solid line. Evolutionary trajectories show that systems become circular long before merger, even for high initial $e$, unless they form with extremely short merger times or extremely high $e$ (see Section 5). For initially circular orbits, $a$ needs to be smaller than $0.215 \mathrm{AU}$ or $46 R_{\odot}$ for the binary to merge within $\sim 10 \mathrm{Gyr}$. BBHs that form from two existing BHs in clusters can appear anywhere in the allowed parameter range. In the case of isolated binaries, the separation before the formation of the second $\mathrm{BH}$ needs to be wide enough to accommodate the progenitor star. The $\mathrm{BBH}$ then forms with a similar separation (or similar periastron distance, if there is mass loss in the $\mathrm{SN}$ or if BHs receive natal kicks), unless the BH kick is large and fine-tuned in its direction to drastically reduce the semimajor axis. Since these progenitor stars have radii of at least several $R_{\odot}\left(\gtrsim 10 R_{\odot}\right.$ for chemically homogeneous evolution), we estimate that the periastron distance needs to be larger than $\sim 10-20 R_{\odot}$ as indicated in Figure 3.

We conclude that, based on published model results, "heavy" BBH mergers from low-metallicity environments in the local universe are not particularly surprising, regardless of whether their origin is dynamical or from isolated binaries. The rate of "heavy" BBH mergers may very well increase with redshift either due to the increase in low metallicity star formation rates or due to higher rates at shorter merger times, at least for redshifts of up to $\simeq 1$. These redshifts are within the horizon distance of aLIGO/Advanced Virgo (AdV) design sensitivity, expected to be reached by $\sim 2020$ (Abbott et al. 2016i, and see Section 7).

\section{BINARY ECCENTRICITY AND BH SPINS}

There is no evidence for eccentricity in the orbital dynamics of GW150914, but eccentricities of $\lesssim 0.1$ would not be detectable for this event (LVC16d). In any case, from Figure 3 it is clear that any eccentricity would have dissipated by the time the binary entered the detectors' sensitive frequency band. Indeed, in this Figure we plot the evolution of a system that would retain an eccentricity of 0.1 at $10 \mathrm{~Hz}$, but this evolution only takes 1.25 days from $e=0.999$ to merger! Kowalska et al. (2011) and Belczynski et al. (2002) show that for their field $\mathrm{BBH}$ models, the expected eccentricities would be undetectable. Only formation in a dynamical environment at a short semimajor axis and an extremely high eccentricity could produce a detectable eccentricity (e.g., O'Leary et al. 2009) for a small fraction of BBHs. A small fraction of BBHs may form through triple stars in globular clusters and potentially maintain significant eccentricities until the merger (Samsing et al. 2014; Antonini et al. 2015).

Parameter-estimation analysis of GW150914 (LVC16d) with gravitational waveforms that account for spin effects (including precession) constrains the dimensionless spin magnitude of the primary $\mathrm{BH}$ to $\lesssim 0.7$ (at $90 \%$ credible level); the spin of the secondary $\mathrm{BH}$ is not significantly constrained. The dimensionless spin components aligned (or anti-aligned) with the orbital angular momentum axis are likely to be small, whereas the spin components in the orbital plane are poorly constrained. The tentative implication is that, if spin magnitudes are indeed large, then the spin-orbit misalignment is likely to be high, too; if the spin magnitudes are small, then the tilts remain unconstrained.

These BH spin magnitude constraints derived from GW observations are comparable in strength to what we typically obtain from X-ray data analyses (for reviews, see McClintock et al. 2014; Miller \& Miller 2015). These BH spin estimates in XRBs have been made from analysis of the X-ray spectra of accretion disks, based on the influence of the $\mathrm{BH}$ spin either on the disk inner edge, which affects the spectral continuum, or on the shape of emission lines. BH spins are typically found to be high for systems with high-mass donors. In general, the cores of massive stars are expected to rotate rapidly and thus may lead to rapid $\mathrm{BH}$ spin at formation, unless there is efficient angular momentum coupling between the core and the (expanding) envelope (e.g., Hirschi et al. 2005). The ability to constrain the $\mathrm{BH}$ spins in GW150914 reveals a new approach to understanding the spin distribution of $\mathrm{BHs}$ that is independent of XRB measurements. Measuring $\mathrm{BH}$ spins in a variety of $\mathrm{BH}$ binaries has the potential of revealing the origin of $\mathrm{BH}$ spins, at formation and through subsequent accretion evolution in binaries (e.g., Belczynski et al. 2008b; Valsecchi et al. 2010; Wong et al. 2012; Amaro-Seoane \& Chen 2015; Fragos \& McClintock 2015).

For $\mathrm{BBH}$ formation from isolated binaries, $\mathrm{BH}$ spin alignment is expected if the spin of the $\mathrm{BH}$ is aligned with the spin of its progenitor star and thus with the binary. Even if $\mathrm{BH}$ kicks are relatively large $\left(>100 \mathrm{~km} \mathrm{~s}^{-1}\right)$, it is found that $\mathrm{BBH}$ spin tilts are rather constrained to typical values below 
about $20^{\circ}$ (Kalogera 2000). For BBH formation in dense environments there is no reasoning suggesting that spins would be correlated in any way through $\mathrm{BH}$ interactions and thus significant misalignment would be more likely. Thus, if we knew that the BH spins in GW150914 were aligned with the orbital angular momentum, then their magnitude would already be constrained by this GW measurement. Conversely, spin precession effects significantly modify the relative orientation of the two BH spins between their formation and merger (Schnittman 2004; Gerosa et al. 2015; Kesden et al. 2015), particularly when both spin magnitudes are large. While initially random spins remain random at coalescence, spin precession effects can distort the relative likelihood of some misalignment angles. Therefore the misalignment angles measured for GW150914 cannot be directly identified as the birth $\mathrm{BH}$ spin misalignments.

We conclude that the non-detection of eccentricity for GW150914 is not a surprise regardless of the BBH formation mechanism. Since the spin magnitude is not expected to change during the XRB phase, the upper limit on the primary BH spin indicates that it was not formed with extremal spin. At present, the evidence for relatively small magnitudes of the $B H$ spin components aligned with the orbital angular momentum does not provide constraints on the formation mechanism. The nonaligned components and hence spin-orbit tilts are essentially unconstrained. With additional BBH detections, a clear preference for mostly aligned spins would favor formation from isolated binaries and small natal BH kicks. On the other hand, a predominance of large misalignments could favor formation through dynamical processes instead. As the sample grows, spin measurements will prove critical for distinguishing formation channels and their relative contributions to the merger rate.

\section{BBH MERGER RATES}

The upper limits on the merger rates from initial LIGO/ Virgo observations were not stringent enough to exclude even the most optimistic theoretical predictions (Aasi et al. 2013b). In contrast, GW150914 provides the first interesting GW rate constraints on astrophysical models. As discussed in Section 2, the rate at which such BBH mergers occur in the low-redshift universe $(z \lesssim 0.5)$ is inferred to be in the range of $2-400 \mathrm{Gpc}^{-3} \mathrm{yr}^{-1}$ (LVC16f).

Over the years, some studies have discussed models of isolated binary populations that result in completely aborting the formation of merging BBH systems (e.g., Nelemans et al. 2001, Belczynski et al. 2002, and most recently Mennekens \& Vanbeveren 2014). In all of these models, the lack of BBH mergers can be traced back to one or more of the following model assumptions: strong (old) wind models, no metallicity dependence of wind strengths, no orbital evolution due to tides, and high BH natal kicks. All of these assumptions effectively widen the orbits of massive binaries and prevent, not the formation of $\mathrm{BBH}$ systems in general, but more specifically the formation of $\mathrm{BBH}$ systems that merge within the age of the universe. Dynamical formation of BBHs is aborted if $\mathrm{BHs}$ receive natal kicks larger than the local escape speed (e.g., $\gtrsim 50 \mathrm{~km} \mathrm{~s}^{-1}$ for typical globular clusters, see Gnedin et al. 2002) such that the BHs escape before they can interact.
The existence of GW150914 shows that BBH mergers occur in nature, and therefore models that do not predict their existence within a Hubble time through any formation channel are excluded (e.g., certain models in Nelemans et al. 2001; Belczynski et al. 2002, 2007; Mennekens \& Vanbeveren 2014). For both isolated binary evolution and dynamical formation, the implication of BBH existence is that BH kicks cannot always be high (>100 $\left.\mathrm{km} \mathrm{s}^{-1}\right)$, in order to avoid disrupting or widening the orbits too much, or ejecting the BHs from clusters before they can interact. In the case of isolated binaries, $B B H$ existence also implies that massive-star winds cannot be strong, and in the absence of high rotation, survival through common-envelope evolution in massive binaries must be possible.

Rate predictions for binary mergers and associated LIGO/ Virgo detection expectations were summarized in Abadie et al. (2010), and for BBH mergers a range of 0.1-300 $\mathrm{Gpc}^{-3} \mathrm{yr}^{-1}$ was reported. More recent studies, not included in Abadie et al. (2010), for isolated binary evolution give very similar predictions: $0-100 \mathrm{Gpc}^{-3} \mathrm{yr}^{-1}$ by Mandel \& de Mink (2016), 0.5-220 Gpc $\mathrm{Gr}^{-3} \mathrm{yr}^{-1}$ by Dominik et al. (2015), and $0-1000 \mathrm{Gpc}^{-3} \mathrm{yr}^{-1}$ by Mennekens \& Vanbeveren (2014). Recent studies of globular cluster dynamics also report comparable rates (Downing et al. 2010, 2011; Rodriguez et al. 2015, 2016). We conclude that the GW150914 rate constraints are broadly consistent with most of the $B B H$ rate predictions, and only the lowest predicted rates $(\lesssim 1$ $\mathrm{Gpc}^{-3} \mathrm{yr}^{-1}$ ) can be excluded.

\section{THE PATH FORWARD FOR FUTURE STUDIES}

In the coming years the aLIGO and AdV detectors will be upgraded to a higher sensitivity, as shown in Figure 4: on the left we plot the maximum luminosity distance $\left(D_{L}\right)$ and redshift $(z)$, and on the right a measure for the surveyed volume $\left(\bar{V}_{c}\right)$ for the initial LIGO/Virgo detectors, the current aLIGO, and future expectations (see the Appendix for the details). We can anticipate that the $\mathrm{BBH}$ detection sample will increase by at least a factor of $\sim 10$ as observing runs become more sensitive and of longer duration. With these new detections, it will become possible to go beyond the mostly qualitative inferences discussed here, and quantitatively constrain the properties of double compact objects (DCOs) and their formation models.

In general, quantitative constraints can be obtained either by deriving a parametrized description of the underlying model (e.g., Mandel 2010; O’Shaughnessy 2013) or by comparing specific population models to the data (e.g., Bulik \& Belczyński 2003; Mandel \& O’Shaughnessy 2010).

For the latter, detailed information about the models and properties of the predicted populations are needed, such as, e.g., masses and rate densities as a function of redshift. Given the large number of model parameters, it is challenging to obtain a statistically appropriate sampling of the parameter space to the level required to address degeneracies; no existing study has provided a sufficiently complete data set. However, such analyses will eventually allow us to constrain massive-star winds and rotation, the common-envelope binary evolution phase, $\mathrm{BH}$ mass relations, and $\mathrm{BH}$ kicks. GW detections of binaries with NSs will probe lower-mass stars as well as NS kicks and the SN mechanism. For dynamical formation, we can also probe cluster properties and their dependence on redshift. 

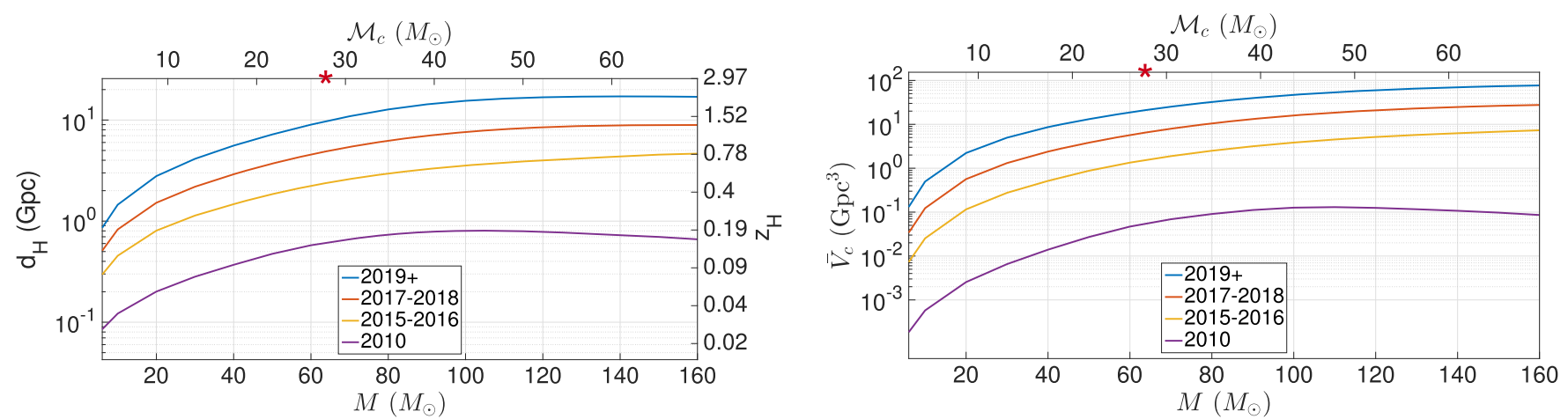

Figure 4. Left: horizon distance (left axis) and horizon redshift (right axis) as a function of total mass (bottom axis) and chirp mass (top axis) for equal-mass, nonspinning BBH mergers. The (expected) increase in detector sensitivity with time is shown by the different lines and the chirp mass of GW150914 is indicated with a red star. Right: the same, but now for detection-weighted sensitive comoving volume, defined to yield the expected number of detections if multiplied with a merger rate per unit volume. For details see the Appendix.

In the past, binary pulsars, $\mathrm{SNe}$, and gamma-ray burst observations have been used as constraints on DCO models (e.g., Portegies Zwart \& Yungelson 1998; O’Shaughnessy et al. 2005b). More recently, studies have explored quantitative, statistical methods for deriving constraints and have examined the minimum sample sizes needed for distinguishing between a small set of different isolated-binary models (Bulik \& Belczyński 2003; Kelley et al. 2010; Mandel \& O’Shaughnessy 2010; Messenger \& Veitch 2013; O’Shaughnessy 2013; Belczynski et al. 2015; Mandel et al. 2015; Stevenson et al. 2015). We note that the majority of these studies conclude that sample sizes of order 100 events are needed for strong constraints.

Before comprehensive quantitative constraints on models become possible, one might consider whether measurements for this one event or just a handful of sources would allow us to distinguish between the two main formation paths: isolated binaries and dynamical processes. The masses of the BHs in $\mathrm{BBH}$ systems from both isolated binary formation and from clusters depend on the mass spectrum of single BHs, and thus in both formation channels a range of masses is expected. For example, the Belczynski et al. (2015) isolated binary models find detectable BBHs with total masses from about 15 to $20 M_{\odot}$ all the way up to $\sim 100 M_{\odot}$ (chirp masses up to $\sim 50 M_{\odot}$ ), the Rodriguez et al. $(2015,2016)$ cluster models find chirp masses of $10-22 M_{\odot}$ (that could be higher for weaker stellar winds), and the Kinugawa et al. (2014) PopIII BBH mergers have higher chirp masses (most above $20 M_{\odot}$ ). The strong dependence on chirp mass of the distance to which sources can be detected (see Figure 4) strongly enhances the probability of detecting these massive $\mathrm{BBHs}$ compared to lower-mass objects (Flanagan \& Hughes 1998).

In view of these predictions, distinguishing between formation in isolated binaries and through dynamical processes based solely on mass measurements is unlikely. The situation is similar for mass ratios: $\mathrm{BBH}$ formation through both isolated binary evolution and dynamical processes tends to favor binaries of roughly comparable masses, within a factor of $\sim 2$ (Dominik et al. 2015; Rodriguez et al. 2015; Mandel \& de Mink 2016).

Initial eccentricities would be very different through the two paths, but most current predictions are for binaries having circularized by the time they enter the frequency band of relevance to ground-based interferometers (see Section 5). An accurate localization of the source would make it possible to check for the presence of nearby clusters. For such localization with GW detectors only, additional advanced detectors and a very high signal-to-noise ratio would be needed. Alternatively, the discovery of an electromagnetic counterpart could pinpoint the position ( $\mathrm{LVC1} 16 \mathrm{~h})$. At present, we are left with two possibilities for distinguishing among formation paths: $\mathrm{BH}$ spins or precise determination of the $\mathrm{BBH}$ merger rate as a function of redshift. Detection of spin misalignment would be a strong indication for dynamical formation, but is challenging, as GW spin measurements are typically not well constrained (e.g., van der Sluys et al. 2008a, 2008b; Vitale et al. 2014; LVC16d); the rates option is challenging too, given the large, overlapping ranges in the rate predictions from the two paths and their uncertain redshift evolution. In the future, we may be able to further constrain models by combining $\mathrm{BBH}$ rate constraints with constraints on NS mergers (even if only upper limits). Consideration of the models consistent with all of these constraints will allow us to make firmer predictions for detection expectations of other types of $\mathrm{EM} / \mathrm{GW}$ binaries involving NSs and white dwarfs.

The BBH population discovered through GW150914 also has implications for other GW detections. First, before entering the aLIGO/AdV band, the BBH systems evolve through the frequency range of space-borne GW detectors such as (e)LISA $(0.1-10 \mathrm{mHz})$ (Amaro-Seoane et al. 2013). Because of the high masses of systems like GW150914, it only takes $\sim 1000$ years to evolve from $2-3 \mathrm{mHz}$ to merger and the systems can be detected not only inside the Milky Way, but to distances of $\sim 10 \mathrm{Mpc}$. These "heavy" stellar-mass BBHs could be plausible (e)LISA sources, if the merger rate is at the upper end of the inferred range.

Second, the expected increase in the merger rate of $\mathrm{BBHs}$ toward higher redshift opens the possibility that the large number of individually unresolvable high-redshift $\mathrm{BBH}$ mergers would instead form a detectable stochastic background signal. Such a signal could be probed with aLIGO/AdV detection of, or upper limits on, the stochastic GW background, as explored in detail in LVC16g.

The possibility that GW150914 is produced by a binary of the first-generation PopIII stars may provide a direct link between the local universe and the BHs that may have been the seeds that grew into the supermassive BHs we find in the centers of most galaxies (Volonteri et al. 2003). Even if GW150914 itself is not a product of PopIII stars, the confirmation of the high $\mathrm{BH}$ masses expected from the weaker stellar winds of low-metallicity stars also supports the idea that PopIII stars may produce even more massive BHs, unless they 
become so massive that they are completely disrupted by pairinstability SNe (e.g., Fryer et al. 2001; Woosley et al. 2007).

\section{CONCLUSIONS}

We have examined the implications of the GW discovery of a BBH merger in the context of the existing literature on the formation of BBHs in isolated binaries and in dense stellar environments. Despite the fact that we have only one firm detection, we can draw several astrophysical conclusions.

For the first time we have observational evidence that BBH systems actually form in nature, with properties such that they merge in the local universe. This is a unique confirmation of numerous theoretical predictions over the past 40 years that merging $\mathrm{BBHs}$ can form, from both isolated binaries in galactic fields and from dense stellar environments. Notably, the measured $\mathrm{BH}$ masses in the merging binary are higher than any of the $\mathrm{BH}$ masses dynamically measured reliably from XRBs. Such "heavy" BHs require that they were formed from massive stars in low-metallicity environments $\left(1 / 2 Z_{\odot}\right.$ or lower), given our current understanding of massive-star winds and their dependence on metallicity. Model rate predictions from both formation mechanisms are broadly consistent with the BBH merger rate implied by the GW150914 discovery. The relatively extreme models that either abort the formation of merging BBHs or predict rates lower than $\simeq 1 \mathrm{Gpc}^{-3} \mathrm{yr}^{-1}$ are now excluded. Apart from weaker winds at low metallicities, a significant fraction of BHs must receive low kicks; survival through common-envelope phases or high rotation in massive stars may be necessary. We note that the majority of recent model predictions survive this constraint. Targeted simulations and additional $\mathrm{GW}$ merger detections will be needed to quantify the balance between $\mathrm{BBH}$ formation rate, delay times until merger, and hence, BBH merger rates as a function of redshift. This first BBH discovery already has implications for a stochastic GW background and for the potential of observations with a future eLISA-like space mission.

These are the key conclusions we can derive based on the GW150914 properties and the existing DCO astrophysics literature. Final analysis of this first aLIGO observational run may provide additional rate constraints from additional detections of BBHs or NS binaries, or in their absence interesting upper limits on merger rates of NS binaries. These combined rate constraints will provide the most stringent quantitative limits on model predictions. An increased source sample resulting from future GW data will of course better constrain the merger rates, but will also allow us to probe the mass distributions and any dependence on redshift. To go beyond the current, mostly qualitative discussion, and move toward comprehensive model constraints, it will be important to develop frameworks that account for observational biases and for appropriate sampling of the model parameter space including relevant parameter degeneracies.

In closing, we are looking forward to the development of $\mathrm{GW}$ astronomy as a new way of probing the universe.

The authors gratefully acknowledge the support of the United States National Science Foundation (NSF) for the construction and operation of the LIGO Laboratory and Advanced LIGO, as well as the Science and Technology Facilities Council (STFC) of the United Kingdom, the MaxPlanck-Society (MPS), and the State of Niedersachsen/ Germany for support of the construction of Advanced LIGO and construction and operation of the GEO600 detector. Additional support for Advanced LIGO was provided by the Australian Research Council. The authors gratefully acknowledge the Italian Istituto Nazionale di Fisica Nucleare (INFN), the French Centre National de la Recherche Scientifique (CNRS), and the Foundation for Fundamental Research on Matter supported by the Netherlands Organisation for Scientific Research for the construction and operation of the Virgo detector and the creation and support of the EGO consortium. The authors also gratefully acknowledge research support from these agencies as well as by the Council of Scientific and Industrial Research of India, Department of Science and Technology, India, Science \& Engineering Research Board (SERB), India, Ministry of Human Resource Development, India, the Spanish Ministerio de Economía y Competitividad, the Conselleria d'Economia i Competitivitat and Conselleria d'Educació Cultura i Universitats of the Govern de les Illes Balears, the National Science Centre of Poland, the European Union, the Royal Society, the Scottish Funding Council, the Scottish Universities Physics Alliance, the Lyon Institute of Origins (LIO), the National Research Foundation of Korea, Industry Canada and the Province of Ontario through the Ministry of Economic Development and Innovation, the National Science and Engineering Research Council Canada, the Brazilian Ministry of Science, Technology, and Innovation, the Leverhulme Trust, the Research Corporation, Ministry of Science and Technology (MOST), Taiwan, and the Kavli Foundation. The authors gratefully acknowledge the support of the NSF, STFC, MPS, INFN, CNRS, and the State of Niedersachsen/Germany for provision of computational resources.

\section{APPENDIX}

The sensitivity of the detector network to GW emission from equal-mass BBHs with non-spinning components is calculated using the following procedure. We use a single-detector signalto-noise ratio threshold of 8 as a proxy for the detectability of binary mergers by a detector network; this is a commonly used proxy (e.g., Abadie et al. 2010) that has been demonstrated to be accurate to within $\sim 10 \%$ for computing surveyed volumes (LVC16f). In Figure 4 the curves labeled 2010, 2015-2016, 2017-2018, and 2019+ are computed using, respectively, the measured noise power spectral density (PSD) of $\mathrm{H} 1$ during the S6 science run, the measured noise PSD of H1 during the 2015 science run, low-end predictions for LIGO noise PSD for the late stages of detector commissioning, and for design sensitivity runs in the zero detuning, high power configuration (Abbott et al. 2016i). We use inspiral-mergerringdown effective one-body waveforms calibrated to numerical relativity for these calculations (Taracchini et al. 2014). The actual sensitivity will depend on the exact network configuration, the data quality, and the signal parameters, so the curves in Figure 4 should be viewed only as approximations. In particular, the signal strength and detectability generally depend on $\mathrm{BH}$ spins.

In Figure 4 the left panel shows the horizon distance, which is the luminosity distance at which GWs from a face-on, equalmass, overhead binary with the given source-frame total mass (bottom axis) or chirp mass (top axis) would be detected at a signal-to-noise ratio of 8; the corresponding redshift is shown on the right vertical axis. The right panel shows the surveyed 
detection-weighted comoving volume $\bar{V}_{c}$

$$
\bar{V}_{c}=\int_{0}^{\infty} \frac{d V_{c}}{d z} f_{d}(z) \frac{1}{1+z} d z,
$$

where $\frac{d V_{c}}{d z}$ is computed using the Planck Collaboration et al. (2015) cosmology and the last factor corrects for the difference in source and observer clocks. Because the GW strength of signals depends (to within factors $\sim 2$ ) on inclination and the detector response depends strongly on sky position, $f_{d}(z)$ is the probability that a binary with the given source-frame masses at redshift $z$ is louder than the signal-to-noise ratio threshold of 8 (integrated over isotropic sky locations and orientations). With this definition, and assuming a constant volumetric merger rate $\mathcal{R}$ per unit comoving volume per unit source time, the expected number of detections during an observing run of duration $T$ is given by $\mathcal{R} \bar{V}_{c} T$.

\section{REFERENCES}

Aasi, J., Abadie, J., Abbott, B. P., et al. 2013, PhRvD, 87, 022002 Abadie, J., Abbott, B. P., Abbott, R., et al. 2010, CQGra, 27, 173001 Abbott, B. P., Abbott, R., Abbott, T. D., et al. 2016a, PhRvL, 116, 061102 (LVC16a)

Abbott, B. P., Abbott, R., Abbott, T. D., et al. 2016b, PhRvD, submitted (LVC16b; https://dcc.ligo.org/LIGO-P1500229/public/main)

Abbott, B. P., Abbott, R., Abbott, T. D., et al. 2016c, PhRvD, submitted (LVC16c; https://dcc.ligo.org/LIGO-P1500269/public/main)

Abbott, B. P., Abbott, R., Abbott, T. D., et al. 2016d, PhRvL, submitted (LVC16d; https://dcc.ligo.org/LIGO-P1500218/public/main)

Abbott, B. P., Abbott, R., Abbott, T. D., et al. 2016e, PhRvL, submitted (LVC16e; https://dcc.ligo.org/LIGO-P1500213/public/main)

Abbott, B. P., Abbott, R., Abbott, T. D., et al. 2016f, ApJL, submitted (LVC16f; https://dcc.ligo.org/LIGO-P1500217/public/main)

Abbott, B. P., Abbott, R., Abbott, T. D., et al. 2016g, PhRvL, submitted (LVC16g; https://dcc.ligo.org/LIGO-P1500222/public/main)

Abbott, B. P., Abbott, R., Abbott, T. D., et al. 2016h, ApJL, submitted (LVC16h; https://dcc.ligo.org/LIGO-P1500227/public/main)

Abbott, B. P., Abbott, R., Abbott, T. D., et al. 2016i, LRR, 19, 1

Amaro-Seoane, P., Aoudia, S., Babak, S., et al. 2013, GWN, 6, 4 Amaro-Seoane, P., \& Chen, X. 2015, arXiv:1512.04897

Antonini, F., Chatterjee, S., Rodriguez, C. L., et al. 2015, arXiv:1509.05080

Bae, Y.-B., Kim, C., \& Lee, H. M. 2014, MNRAS, 440, 2714

Banerjee, S., Baumgardt, H., \& Kroupa, P. 2010, MNRAS, 402, 371

Belczynski, K., Bulik, T., Fryer, C. L., et al. 2010a, ApJ, 714, 1217

Belczynski, K., Bulik, T., \& Rudak, B. 2004, ApJL, 608, L45

Belczynski, K., Buonanno, A., Cantiello, M., et al. 2014, ApJ, 789, 120

Belczynski, K., Dominik, M., Bulik, T., et al. 2010b, ApJL, 715, L138

Belczynski, K., Kalogera, V., \& Bulik, T. 2002, ApJ, 572, 407

Belczynski, K., Kalogera, V., Rasio, F. A., et al. 2008a, ApJS, 174, 223

Belczynski, K., Repetto, S., Holz, D., et al. 2015, arXiv:1510.04615

Belczynski, K., Taam, R. E., Kalogera, V., Rasio, F. A., \& Bulik, T. 2007, ApJ, 662, 504

Belczynski, K., Taam, R. E., Rantsiou, E., \& van der Sluys, M. 2008b, ApJ, 682,474

Benacquista, M. J., \& Downing, J. M. B. 2013, LRR, 16, 4

Bolton, C. T. 1972, Natur, 235, 271

Bulik, T., \& Belczyński, K. 2003, ApJL, 589, L37

Bulik, T., Belczynski, K., \& Prestwich, A. 2011, ApJ, 730, 140

Casares, J., \& Jonker, P. G. 2014, SSRv, 183, 223

Chomiuk, L., Strader, J., Maccarone, T. J., et al. 2013, ApJ, 777, 69

Crowther, P. A., Barnard, R., Carpano, S., et al. 2010, MNRAS, 403, L41

de Mink, S. E., \& Belczynski, K. 2015, ApJ, 814, 58

de Mink, S. E., Cantiello, M., Langer, N., et al. 2009, A\&A, 497, 243

Dewey, R. J., \& Cordes, J. M. 1987, ApJ, 321, 780

Dominik, M., Belczynski, K., Fryer, C., et al. 2012, ApJ, 759, 52

Dominik, M., Belczynski, K., Fryer, C., et al. 2013, ApJ, 779, 72

Dominik, M., Berti, E., O’Shaughnessy, R., et al. 2015, ApJ, 806, 263

Downing, J. M. B., Benacquista, M. J., Giersz, M., \& Spurzem, R. 2010, MNRAS, 407, 1946
Downing, J. M. B., Benacquista, M. J., Giersz, M., \& Spurzem, R. 2011, MNRAS, 416, 133

Farr, W. M., Gair, J. R., Mandel, I., \& Cutler, C. 2015, PhRvD, 91, 023005

Farr, W. M., Sravan, N., Cantrell, A., et al. 2011, ApJ, 741, 103

Flanagan, E. E., \& Hughes, S. A. 1998, PhRvD, 57, 4535

Fragos, T., \& McClintock, J. E. 2015, ApJ, 800, 17

Fryer, C. L., Belczynski, K., Wiktorowicz, G., et al. 2012, ApJ, 749, 91

Fryer, C. L., \& Kalogera, V. 2001, ApJ, 554, 548

Fryer, C. L., Woosley, S. E., \& Heger, A. 2001, ApJ, 550, 372

Gallazzi, A., Charlot, S., Brinchmann, J., White, S. D. M., \& Tremonti, C. A 2005, MNRAS, 362, 41

Gerosa, D., Kesden, M., O’Shaughnessy, R., et al. 2015, PhRvL, 115, 141102

Glebbeek, E., Gaburov, E., de Mink, S. E., Pols, O. R., \& Portegies Zwart, S. F. 2009, A\&A, 497, 255

Gnedin, O. Y., Zhao, H., Pringle, J. E., et al. 2002, ApJL, 568, L23

Goswami, S., Kiel, P., \& Rasio, F. A. 2014, ApJ, 781, 81

Gültekin, K., Miller, M. C., \& Hamilton, D. P. 2004, ApJ, 616, 221

Gültekin, K., Miller, M. C., \& Hamilton, D. P. 2006, ApJ, 640, 156

Hannam, M., Schmidt, P., Bohé, A., et al. 2014, PhRvL, 113, 151101

Heger, A., Fryer, C. L., Woosley, S. E., Langer, N., \& Hartmann, D. H. 2003, ApJ, 591, 288

Heggie, D. C. 1975, MNRAS, 173, 729

Hirschi, R., Meynet, G., \& Maeder, A. 2005, A\&A, 443, 581

Hulse, R. A., \& Taylor, J. H. 1975, ApJL, 195, L51

Jones, S., Hirschi, R., Pignatari, M., et al. 2015, MNRAS, 447, 3115

Kalogera, V. 2000, ApJ, 541, 319

Kalogera, V., \& Baym, G. 1996, ApJL, 470, L61

Kalogera, V., Belczynski, K., Kim, C., O’Shaughnessy, R., \& Willems, B. 2007, PhR, 442, 75

Kelley, L. Z., Ramirez-Ruiz, E., Zemp, M., Diemand, J., \& Mandel, I. 2010, ApJL, 725, L91

Kesden, M., Gerosa, D., O’Shaughnessy, R., Berti, E., \& Sperhake, U. 2015, PhRvL, 114, 081103

Khan, S., Husa, S., Hannam, M., et al. 2015, arXiv:1508.07253

Kim, C., Kalogera, V., \& Lorimer, D. R. 2003, ApJ, 584, 985

Kinugawa, T., Inayoshi, K., Hotokezaka, K., Nakauchi, D., \& Nakamura, T. 2014, MNRAS, 442, 2963

Kobulnicky, H. A., \& Fryer, C. L. 2007, ApJ, 670, 747

Kobulnicky, H. A., Kiminki, D. C., Lundquist, M. J., et al. 2014, ApJS, 213, 34

Kocsis, B., Gáspár, M. E., \& Márka, S. 2006, ApJ, 648, 411

Kocsis, B., \& Levin, J. 2012, PhRvD, 85, 123005

Kormendy, J., \& Richstone, D. 1995, ARA\&A, 33, 581

Kornilov, V. G., \& Lipunov, V. M. 1983, SvA, 27, 334

Kowalska, I., Bulik, T., Belczynski, K., Dominik, M., \& Gondek-Rosinska, D. 2011, A\&A, 527, A70

Kulkarni, S. R., Hut, P., \& McMillan, S. 1993, Natur, 364, 421

Laycock, S. G. T., Maccarone, T. J., \& Christodoulou, D. M. 2015, MNRAS, 452, L31

Lipunov, V. M., Postnov, K. A., \& Prokhorov, M. E. 1997, MNRAS, 288, 245

Maccarone, T. J., Kundu, A., Zepf, S. E., \& Rhode, K. L. 2007, Natur, 445, 183

Madau, P., \& Dickinson, M. 2014, ARA\&A, 52, 415

Maeder, A. 1987, A\&A, 178, 159

Maeder, A. 1992, A\&A, 264, 105

Mandel, I. 2010, PhRvD, D81, 084029

Mandel, I., \& de Mink, S. E. 2016, arXiv:1601.00007

Mandel, I., Haster, C.-J., Dominik, M., \& Belczynski, K. 2015, MNRAS, 450, L85

Mandel, I., \& O'Shaughnessy, R. 2010, CQGra, 27, 114007

Mapelli, M., Colpi, M., \& Zampieri, L. 2009, MNRAS, 395, L71

Mapelli, M., \& Zampieri, L. 2014, ApJ, 794, 7

Mapelli, M., Zampieri, L., Ripamonti, E., \& Bressan, A. 2013, MNRAS, 429, 2298

Marchant, P., Langer, N., Podsiadlowski, P., Tauris, T., \& Moriya, T. 2016, arXiv: 1601.03718

McClintock, J. E., Narayan, R., \& Steiner, J. F. 2014, SSRv, 183, 295

McClintock, J. E., \& Remillard, R. A. 2006, in Compact Stellar X-Ray Sources, ed. W. Lewin, \& M. van der Klis (Cambridge: Cambridge Univ. Press), 157

Mennekens, N., \& Vanbeveren, D. 2014, A\&A, 564, A134

Messenger, C., \& Veitch, J. 2013, NJPh, 15, 053027

Miller, M. C., \& Lauburg, V. M. 2009, ApJ, 692, 917

Miller, M. C., \& Miller, J. M. 2015, PhR, 548, 1

Morscher, M., Pattabiraman, B., Rodriguez, C., Rasio, F. A., \& Umbreit, S. 2015, ApJ, 800, 9

Morscher, M., Umbreit, S., Farr, W. M., \& Rasio, F. A. 2013, ApJL, 763, L15 
Nelemans, G., Yungelson, L. R., \& Portegies Zwart, S. F. 2001, A\&A, 375, 890

O’Leary, R. M., Kocsis, B., \& Loeb, A. 2009, MNRAS, 395, 2127

O’Leary, R. M., O’Shaughnessy, R., \& Rasio, F. A. 2007, PhRvD, 76, 061504

O’Leary, R. M., Rasio, F. A., Fregeau, J. M., Ivanova, N., \& O'Shaughnessy, R. 2006, ApJ, 637, 937

O’Shaughnessy, R. 2013, PhRvD, 88, 084061

O’Shaughnessy, R., Kaplan, J., Kalogera, V., \& Belczynski, K. 2005a, ApJ, 632,1035

O’Shaughnessy, R., Kim, C., Fragos, T., Kalogera, V., \& Belczynski, K. 2005b, ApJ, 633, 1076

Özel, F., Psaltis, D., Narayan, R., \& McClintock, J. E. 2010, ApJ, 725, 1918

Peters, P. C. 1964, PhRv, 136, B1224

Planck Collaboration, Ade, P. A. R., Aghanim, N., et al. 2015, arXiv: 1502.01589

Portegies Zwart, S. F., Makino, J., McMillan, S. L. W., \& Hut, P. 1999, A\&A, 348,117

Portegies Zwart, S. F., \& McMillan, S. L. W. 2000, ApJL, 528, L17

Portegies Zwart, S. F., \& Yungelson, L. R. 1998, A\&A, 332, 173

Postnov, K. A., \& Yungelson, L. R. 2014, LRR, 17, 3

Prestwich, A. H., Kilgard, R., Crowther, P. A., et al. 2007, ApJL, 669, L21

Pürrer, M. 2014, CQGra, 31, 195010

Rhoades, C. E., \& Ruffini, R. 1974, PhRvL, 32, 324

Rodriguez, C. L., Morscher, M., Pattabiraman, B., et al. 2015, PhRvL, 115 051101

Rodriguez, C. L., Morscher, M., Pattabiraman, B., et al. 2016, PhRvL, 116 029901

Sadowski, A., Belczynski, K., Bulik, T., et al. 2008, ApJ, 676, 1162

Samsing, J., MacLeod, M., \& Ramirez-Ruiz, E. 2014, ApJ, 784, 71

Sana, H., de Mink, S. E., de Koter, A., et al. 2012, Sci, 337, 444

Schnittman, J. D. 2004, PhRvD, 70, 124020
Schutz, B. F. 1989, CQGra, 6, 1761

Sigurdsson, S., \& Hernquist, L. 1993, Natur, 364, 423

Silverman, J. M., \& Filippenko, A. V. 2008, ApJL, 678, L17

Smith, N. 2014, ARA\&A, 52, 487

Spera, M., Mapelli, M., \& Bressan, A. 2015, MNRAS, 451, 4086

Stevenson, S., Ohme, F., \& Fairhurst, S. 2015, ApJ, 810, 58

Taracchini, A., Buonanno, A., Pan, Y., et al. 2014, PhRvD, 89, 061502

Taylor, J. H., \& Weisberg, J. M. 1982, ApJ, 253, 908

Thorne, K. S. 1987, in Three Hundred Years of Gravitation, ed. S. W. Hawking, \& W. Israel (Cambridge: Cambridge Univ. Press), 330

Tremonti, C. A., Heckman, T. M., Kauffmann, G., et al. 2004, ApJ, 613,898

Tsang, D. 2013, ApJ, 777, 103

Tutukov, A., \& Yungelson, L. 1973, NInfo, 27, 70

Tutukov, A. V., \& Yungelson, L. R. 1993, MNRAS, 260, 675

Ugliano, M., Janka, H.-T., Marek, A., \& Arcones, A. 2012, ApJ, 757, 69

Valsecchi, F., Glebbeek, E., Farr, W. M., et al. 2010, Natur, 468, 77

van der Sluys, M., Raymond, V., Mandel, I., et al. 2008a, CQGra, 25, 184011

van der Sluys, M. V., Röver, C., Stroeer, A., et al. 2008b, ApJL, 688, L61

Vanbeveren, D. 2009, NewA, 53, 27

Vink, J. S. 2008, NewAR, 52, 419

Vitale, S., Lynch, R., Veitch, J., Raymond, V., \& Sturani, R. 2014, PhRvL, 112,251101

Volonteri, M. 2010, A\&ARv, 18, 279

Volonteri, M., Haardt, F., \& Madau, P. 2003, ApJ, 582, 559

Webster, B. L., \& Murdin, P. 1972, Natur, 235, 37

Wong, T.-W., Valsecchi, F., Fragos, T., \& Kalogera, V. 2012, ApJ, 747, 111

Woosley, S. E., Blinnikov, S., \& Heger, A. 2007, Natur, 450, 390

Ziosi, B. M., Mapelli, M., Branchesi, M., \& Tormen, G. 2014, MNRAS, 441, 3703

Zlochower, Y., \& Lousto, C. O. 2015, PhRvD, 92, 024022 